\title{
Invariant manifolds and the response of spiral arms in barred galaxies
}

\author{
P. Tsoutsis ${ }^{1,2}$, C. Kalapotharakos ${ }^{1}$, C. Efthymiopoulos ${ }^{1}$, and G. Contopoulos ${ }^{1}$
}

\begin{abstract}
1 Research Center for Astronomy, Academy of Athens, Soranou Efessiou 4, 11527 Athens, Greece e-mail: [ptsoutsi;ckalapot] @phys.uoa.gr; [cefthim;gcontop]@academyofathens.gr

2 Department of Physics, University of Athens, 11527 Athens, Greece
\end{abstract}

Received 7 May 2008 / Accepted 4 December 2008

\section{ABSTRACT}

\begin{abstract}
The unstable invariant manifolds of the short-period family of periodic orbits around the unstable Lagrangian points $L_{1}$ and $L_{2}$ of a barred galaxy define loci in the configuration space, which take the form of a trailing spiral pattern. In previous works we explored the association of such a pattern to the observed spiral pattern in $N$-body models of barred-spiral galaxies and found it to be quite relevant. Our aims in the present paper are: a) to investigate this association in the case of the self-consistent models of Kaufmann \& Contopoulos (1996, A\&A, 309, 381), which provide an approximation of real barred-spiral galaxies; b) to examine the dynamical role played by each of the non-axisymmetric components of the potential, i.e. the bar and the spiral perturbation, and their consequences on the form of the invariant manifolds; and c) to examine the relation of "response" models of barred-spiral galaxies with the theory of the invariant manifolds. Our method relies on calculating the invariant manifolds for values of the Jacobi constant close to its value for $L_{1}$ and $L_{2}$. Our main results are the following. a) The invariant manifolds yield the correct form of the imposed spiral pattern provided that their calculation is done with the spiral potential term turned on. We provide a theoretical model explaining the form of the invariant manifolds that supports the spiral structure. The azimuthal displacement of the Lagrangian points with respect to the bar's major axis is a crucial parameter in this modeling. When this is taken into account, the manifolds necessarily develop in a spiral-like domain of the configuration space, delimited from below by the boundary of a banana-like non-permitted domain, and from above either by rotational KAM tori or by cantori forming a stickiness zone. On the contrary, if the whole non-axisymmetric perturbation is artificially "aligned" with the bar (i.e. there is no azimuthal shift of the Lagrangian manifolds), the manifolds support a ring rather than a spiral structure. b) We construct "spiral response" models on the basis of the theory of the invariant manifolds and examine the connection of the latter to the "response" models (Patsis 2006, MNRAS, 369, 56) used to fit real barred-spiral galaxies, explaining how the manifolds are related to a number of morphological features seen in such models.
\end{abstract}

Key words. chaos - galaxies: kinematics and dynamics - galaxies: spiral

\section{Introduction}

The ordered or chaotic nature of orbits in barred galaxies has been the subject of many investigations in the literature (Contopoulos 1981; Pfenniger 1984; Sparke \& Sellwood 1987; Pfenniger \& Frendli 1991; Kaufmann \& Contopoulos 1996; Patsis et al. 1997; Fux 2001; Pichardo et al. 2004; Kaufmann \& Patsis 2005). Interest in this problem stems from the fact that the existence (and degree) of chaos has direct consequences on the morphological features of a rotating galaxy. In particular, the appearance of a high degree of chaos in the corotation region is one of the main reasons for the bars terminating near corotation (Contopoulos 1981; see Contopoulos 2002; pp. 473, 474 for a review).

Beyond corotation, prominent structures are commonly observed such as rings or spiral arms. The role of the chaotic orbits in the dynamics of such structures is still a widely open problem, but recently some progress has been made towards its understanding. In particular, a theoretical model has been proposed and numerically explored (Voglis et al. 2006a,b; Romero-Gomez et al. 2006, 2007), according to which the spiral arms (or rings) are supported by the unstable invariant manifolds of the two short-period families of unstable periodic orbits around the unstable Lagrangian equilibria $L_{1}$ and $L_{2}$ (called hereafter the PL1 and PL2 families respectively). This theory was extended by
Tsoutsis et al. (2008), by examining the contribution of the unstable manifolds of other families, besides PL1 or PL2, to the same phenomenon. The importance of the chaotic orbits in supporting the spiral structure of barred galaxies has also been emphasized by Patsis (2006).

The following is a brief account of the theory of the invariant manifolds:

1. We consider a 2D approximation of the orbits in the disk plane of a barred - spiral galaxy, given by the Hamiltonian

$H\left(r, \theta, p_{r}, p_{\theta}\right) \equiv \frac{1}{2}\left(p_{r}^{2}+\frac{p_{\theta}^{2}}{r^{2}}\right)-\Omega_{\mathrm{p}} p_{\theta}+V_{0}(r)+V_{1}(r, \theta)=E_{\mathrm{J}} .(1)$

In this expression, $(r, \theta)$ are polar coordinates in the rotating frame, $p_{r}=\dot{r}, p_{\theta}=r^{2}\left(\dot{\theta}+\Omega_{\mathrm{p}}\right)$ is the angular momentum in the rest frame, $V_{0}$ is the axisymmetric potential and $V_{1}$ is the non-axisymmetric potential perturbation due to the bar and to the spiral arms. The parameter $\Omega_{\mathrm{p}}$ is the angular speed of the rotating frame, which coincides with the bar-spiral pattern speed in an approximation in which the latter is assumed to be unique.

2. The Hamiltonian flow under (1) yields two stable $\left(L_{4}, L_{5}\right)$ and two unstable $\left(L_{1}, L_{2}\right)$ Lagrangian equilibrium points (in the rotating frame) at which a star corotates with the pattern. The unstable manifold $\mathcal{W}_{L_{1}}^{U}$ of $L_{1}$ is defined as the set of all 
(a)

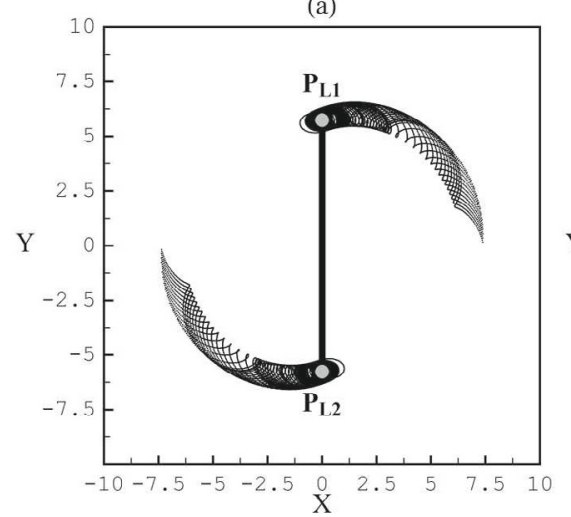

(b)

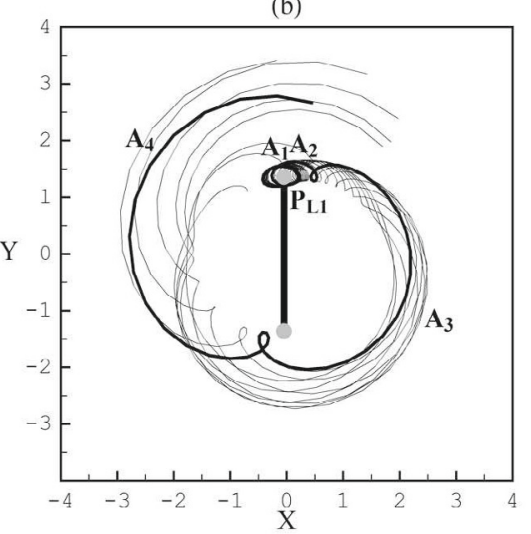

(c)

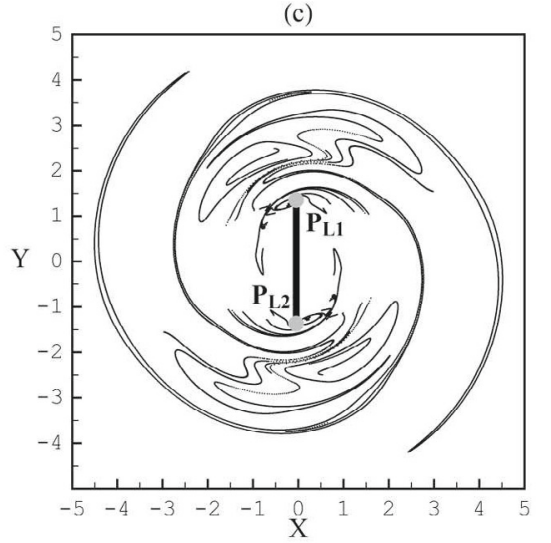

Fig. 1. a) Projections of the invariant manifolds $\mathcal{W}_{\mathrm{PL} 1}^{U}$ and $\mathcal{W}_{\mathrm{PL} 2}^{U}$ on the configuration space, approximated by an ensemble of 20 orbits with initial conditions on the unstable direction of the tangent space to the periodic orbits PL1 and PL2 (small bold circles) in the neighborhood of $L_{1}$ and $L_{2}$. The potential used is from model A (see Sect. 2). The bar is aligned to the $y$-axis and rotates counterclockwise. b) Same as in a) but in a strongly non-linear model (in this case we use the potential of the $N$-Body simulation analyzed in Voglis et al. 2006a. Only the manifold $W_{\mathrm{PL} 1}^{U}$ is plotted). c) The projection of the intersection of the manifolds $\mathcal{W}_{\mathrm{PL} 1}^{U}$ and $\mathcal{W}_{\mathrm{PL} 2}^{U}$ with the apocentric surface of section $p_{r}=0$, $\dot{p}_{r}<0$ on the configuration space of the model of $\mathbf{b})$.

the initial conditions $\left(r_{0}, \theta_{0}, p_{r 0}, p_{\theta 0}\right)$ in the phase space for which the resulting orbit tends asymptotically to $L_{1}$ in the backward sense of time, namely

$$
\begin{aligned}
\mathcal{W}_{L_{1}}^{U}= & \left\{\bigcup\left(r_{0}, \theta_{0}, p_{r 0}, p_{\theta 0}\right):\right. \\
& \left.\lim _{t \rightarrow-\infty}\left\|Q\left(t ; r_{0}, \theta_{0}, p_{r 0}, p_{\theta 0}\right)-\left(r_{L_{1}}, \theta_{L_{1}}, 0, \Omega_{\mathrm{p}}\right)\right\|=0\right\}
\end{aligned}
$$

where $Q\left(t ; r_{0}, \theta_{0}, p_{r 0}, p_{\theta 0}\right)$ denotes the position (point in phase space) at time $t$ of a particle along an orbit starting with the above initial conditions, and the norm $\|\cdot\|$ means the Euclidean distance between this point and the phase space point $\left(r_{L_{1}}, \theta_{L_{1}}, 0, \Omega_{\mathrm{p}}\right)$, corresponding to $L_{1}$. All the points of the manifold $W_{L_{1}}^{U}$ yield the same value of the Jacobi constant, equal to $E_{\mathrm{J}}=E_{\mathrm{J}, L 1}$. Furthermore, since $L_{1}$ is simply unstable, $\mathcal{W}_{L_{1}}^{U}$ is a two-dimensional manifold embedded in the three-dimensional hypersurface of the phase space corresponding to a fixed Jacobi constant $E_{\mathrm{J}}=E_{\mathrm{J}, L 1}$. Similar definitions and properties hold for $L_{2}$ and $\mathcal{W}_{L_{2}}^{U}$ and for the stable manifolds $W_{L_{1}}^{S}, W_{L_{2}}^{S}$, i.e. the sets of initial conditions tending asymptotically to $L_{1}$, or $L_{2}$ in the forward sense of time, as $t \rightarrow \infty$.

3. For $E_{\mathrm{J}}>E_{\mathrm{J}, L 1}$, a short-period unstable periodic orbit (PL1) bifurcates from $L_{1}$ (and the symmetric orbit PL2 from $L_{2}$ ). This orbit forms a small loop around $L_{1}$ (Fig. 1a, thick solid curve), which corresponds to a 1D-torus in the phase space. This torus is "whiskered", i.e., it possesses its own asymptotic manifolds. In particular, the unstable manifold of PL1 is now defined as

$$
\begin{aligned}
\mathcal{W}_{\mathrm{PL} 1}^{U}= & \left\{\bigcup\left(r_{0}, \theta_{0}, p_{r 0}, p_{\theta 0}\right):\right. \\
& \left.\lim _{t \rightarrow-\infty}\left\|Q\left(t ; r_{0}, \theta_{0}, p_{r 0}, p_{\theta 0}\right)-P L 1\right\|=0\right\}
\end{aligned}
$$

where the notation $\|\cdot\|$ refers to the minimum of the distances of $Q(t)$ from the locus of all the phase space points of the orbit PL1. For any fixed value of $E_{\mathrm{J}}>E_{\mathrm{J}, L 1}, W_{\mathrm{PL} 1}^{U}$ is a twodimensional manifold embedded in the three-dimensional hypersurface of constant $E_{\mathrm{J}}$. Figure 1 a shows the projection of a small part of this manifold, close to PL1, in the configuration space $x=r \cos \theta, y=r \sin \theta$. This is drawn approximately, by calculating a number of orbits with initial conditions on $\mathcal{W}_{\mathrm{PL} 1}^{U}$, and close to PL1. The possibility to find such initial conditions is guaranteed by the fact that the manifold $\mathcal{W}_{\text {PL1 }}^{U}$ is tangent to the unstable manifold of the linearized Hamiltonian flow near PL1 (the so-called Grobman 1959; and Hartman 1960 theorem), and the latter is calculated by diagonalizing the Floquet matrix of the orbit PL1. In Fig. 1a we draw the part of the manifold lying outside corotation for a particular model of barred galaxy. We can see that close to PL1 the orbits form epicyclic loops of size nearly equal to the PL1 loop, while, in the same time, the guiding center recedes from PL1 along a path which yields a trailing spiral arm. An analysis of the linearized flow yields that the deviation of the guiding center from PL1 is exponential in time, with a rate determined by the positive characteristic exponents of the Floquet matrix of PL1. Furthermore, in generic galactic potentials all the orbits on $\mathcal{W}_{\mathrm{PL} 1}^{U}$ are chaotic. (The same phenomena hold for the orbit PL2 and the manifold $\mathcal{W}_{\mathrm{PL} 2}^{U}$ also plotted in Fig. 1a).

4. In strongly nonlinear models (as is the case of strongly barred galaxies with conspicuous spiral arms), further away from PL1 the size of the epicycles becomes great (it may exceed the size of the bar). Such an example is shown in Fig. $1 b$, referring to the orbits of the $\mathcal{W}_{\mathrm{PL} 1}^{U}$ family in a $N$-Body model of a barred galaxy (Voglis et al. 2006a,b). We see that one such orbit (bold) forms two relatively small loops near PL1, reaching the apocentric positions $A_{1}$ and $A_{2}$, but the exponential recession of the guiding center is so fast that there is no loop formed between the second and third $\left(A_{3}\right)$ apocentric positions. Furthermore, the fourth apocentric position is at a distance about twice the bar's major semi-axis. Further integration beyond that of Fig. 1b shows that, in fact, all these orbits belong to the so-called "hot population" (Sparke \& Sellwood 1987), i.e., the orbits make several consecutive oscillations in and out of corotation. Kaufmann \& Contopoulos (1996, their Fig. 21a) suggested that such orbits can partly support the bar and partly the spiral arms.

To understand how the chaotic orbits may establish a longtime flow supporting the spiral structure, Voglis et al. (2006a) 
examined a particular subset of points of the unstable manifold $\mathcal{W}_{\mathrm{PL} 1}^{U}$, namely the locus of all apocentric positions of the orbits on $\mathcal{W}_{\mathrm{PL} 1}^{U}$. This is defined by taking the intersection of $\mathcal{W}_{\mathrm{PL} 1}^{U}$ with the so-called surface of section of the apocentric positions on a hypersurface of constant $E_{\mathrm{J}}$, defined as the set of phase space points satisfying $p_{r}=0, \dot{p}_{r}<0$. We stress that the choice of such a surface of section proves to be very relevant to the particular type of study undertaken here. Indeed, whenever $p_{r}=0$, Eq. (1) yields points $\left(r, \theta, p_{\theta}\right)$ which can be projected either on the $\left(\theta, p_{\theta}\right)$ plane (called the "phase portrait" by virtue of the fact that the variables $\left(\theta, p_{\theta}\right)$ are canonically conjugated), or the plane $(\theta, r)$, i.e. the usual plane of motion in the rotating frame. However, in typical galactic potentials the variables $r$ and $p_{\theta}$ at the apocenters have a monotonic relation, and this implies that the phase portraits and $(r, \theta)$ portraits for this particular type of surface of section are isomorphic. This allows us to unravel immediately the consequences of the phase space dynamical features, as seen in the phase portraits, to the morphological features of the system, as seen in the usual disk plane of motion with coordinates $(x, y)=(r \cos \theta, r \sin \theta)$. Such a comparison would not be possible by the use of a traditional surface of section, such as $(x, \dot{x})$ for $y=0, \dot{y}>0$ (or $\dot{y}<0)$.

Returning to the role of the invariant manifolds, the intersection of $\mathcal{W}_{\mathrm{PL} 1}^{U}$ with the apocentric surface of section yields an one-dimensional locus of points. Such a locus can be projected on either the phase portrait plane $\left(\theta, p_{\theta}\right)$ or the configuration space $(r, \theta)$. Figure 1c shows the latter projection in the case of the same manifold as in Fig. 1b, but calculated for a much larger length. Every point in Fig. 1c corresponds to one apocentric position of a chaotic orbit with initial conditions on the unstable manifold. Clearly, the apocentric positions along $\mathcal{W}_{\mathrm{PL} 1}^{U}$ yield a locus which also supports a trailing spiral arm over, however, a much larger extent of $\mathcal{W}_{\mathrm{PL} 1}^{U}$ than in the case of Fig. $1 \mathrm{~b}$. The manifold of Fig. 1c takes a typical form known in dynamical systems' theory to be associated with the so-called phenomenon of homoclinic chaos. Briefly, the manifold develops lobes forming oscillations close to the apocentric points of the periodic orbits PL1 or PL2. Such oscillations are analyzed in detail in the sequel.

We should stress that an analysis of the Floquet matrix of the PL1 or PL2 families yields that only the directions of the unstable invariant manifolds $\mathcal{W}_{\mathrm{PL} 1}^{U}, \mathcal{W}_{\mathrm{PL} 2}^{U}$ are such as to define trailing spiral arms, while, close to $L_{1}$ or $L_{2}$, the stable manifolds $\mathcal{W}_{\mathrm{PL} 1}^{S}, \mathcal{W}_{\mathrm{PL} 2}^{S}$ define leading spiral arms. Furthermore, in the forward sense of time the chaotic orbits are attracted in directions of the phase space along the unstable manifolds. In the sequel we no longer refer to the stable manifolds $W_{\mathrm{PL} 1}^{S}, W_{\mathrm{PL} 2}^{S}$, and the term "invariant manifolds" always implies the unstable manifolds $\mathcal{W}_{\mathrm{PL} 1}^{U}, \mathcal{W}_{\mathrm{PL} 2}^{U}$.

In summary, the theory of the invariant manifolds, viewed as either the loci on which lies the continuous flow of a swarm of orbits (Romero-Gomez et al. 2006, 2007), or the loci of apocentric positions of these orbits (Voglis et al. 2006a; Tsoutsis et al. 2008), predicts the formation by the manifolds of a trailing spiral pattern beyond corotation. Naturally, the central question that should be posed now is whether (and up to what extent) the spiral arms formed self-consistently in real galaxies can be associated with the spiral patterns formed by the invariant manifolds $W_{\mathrm{PL1}, 2}^{U}$. In our previous works (Voglis et al. 2006a; Tsoutsis et al. 2008), we examined this question by considering the spiral arms formed in an $N$-Body model of a barred galaxy and found such an association to be quite relevant.
In the present paper, our main goal is to examine the same question in simple models of real barred-spiral galaxies for which some reliable estimation of both the gravitational potential and the pattern speed have been provided in the literature by methods independent of the previous considerations. To this end, we selected the potential models and pattern speeds reported in the study of Kaufmann \& Contopoulos (1996) for three real galaxies, NGC 3992, NGC 1073 and NGC 1398. This choice is motivated by the fact that Kaufmann \& Contopoulos (1996) constructed approximate self-consistent models of the studied galaxies based on the response density of the superposition of many stellar dynamical orbits. Thus, their study yielded not only plausible values of the potential parameters, or the pattern speed, but also the decomposition of the potential into components, i.e., $V_{\text {halo }}, V_{\text {disk }}, V_{\text {bar }}$ and $V_{\text {spiral }}$. This allows us to check the role of each of these components, in particular of the non-axisymmetric ones $V_{\text {bar }}$ and $V_{\text {spiral }}$, in the theory. It should be noted that the self-consistent technique, pioneered by Schwarzschild (1979), has been used extensively to provide reliable models of galaxies, despite the fact that there is no a priori guarantee of the stability of such models that should ideally be probed via $N$-body simulations (see e.g. Smith \& Miller 1982).

Besides re-confirming that the invariant manifolds do correlate well with the spiral arms found in the self-consistent models of Kaufmann \& Contopoulos (1996), our investigation led to a second non-trivial result analyzed in detail in the sequel: in all three models the bar component is dominant over the spiral component within a large radial extent, but not in a narrow zone beyond corotation. This implies that if one uses only the bar component to calculate the manifolds, the latter yield ring rather than spiral structures. Furthermore, if one adds the spiral perturbation to the potential, but gives no azimuthal tilting to the associated $m=2$ Fourier component, the manifolds become more open as regards their radial extent, but remain quite symmetric as regards their orientation with respect to the bar's major axis, thus still defining rings rather than spiral arms. Only when the azimuthal deformation of the equipotential surfaces due to a really spiral-like perturbation is taken into account (in the Kaufmann \& Contopoulos 1996 paper this was modeled as a simple logarithmic spiral), the manifolds are found to follow closely the spiral arms of the self-consistent models. In some numerical experiments (see Sect. 3 below) we managed to obtain a kind of spiral pattern formed by the initial segments of the invariant manifolds in pure bar models, having, however, to drastically depart from the bar parameters given in Kaufmann and Contopoulos' self-consistent models, and pushing the bar's amplitude to highly non-physical values. But even in that case, the manifold-induced spiral arms are quite different from the spiral arms of the self-consistent models, and they disappear when the manifolds are computed for a longer length. Such an investigation demonstrates that while in principle the strength of the quadrupole moment of the bar's potential causes a "thickening" of ring structures, thus facilitating the phenomenon of appearance of spiral arms (Romero-Gomez et al. 2007), this parameter is not sufficient in order to characterize this phenomenon. The azimuthal displacement of the Lagrangian points is the most important parameter. This result probably provides a dynamical basis for understanding the reported failure of pure bar models to reproduce the inner spiral arms emanating at the ends of bars in both particle and hydrodynamical simulations of barred galaxies (e.g. Lindblad et al. 1996; Aguerri et al. 2001).

The paper is organized as follows: Sect. 2 gives the form of the invariant manifolds in the models of Kaufmann and Contopoulos. We examine the manifolds a) when the spiral 
perturbation is turned-on, and b) in an "aligned model" version in which the whole non-axisymmetric perturbation is artificially aligned to the bar. In case (a) the manifolds yield a spiral response, while in case (b) they yield a ring-like response. Since in all the above models the spiral perturbation is strong, we also examine two models corresponding to a "mean" and "weak" spiral amplitude, created by suitably varying the parameters of some of the original models of Kaufmann \& Contopoulos (1996). We finally provide a theoretical justification of the importance of the azimuthal displacement of the Lagrangian points in the form of the invariant manifolds. Section 3 discusses the connection between the theory of the invariant manifolds and the "spiral response" models constructed via iterative methods. In particular, we propose a method of constructing response models on the basis of populating by matter the manifolds generated by a "pure bar" model. We also calculate response models via the method proposed by Patsis (2006) and discuss a number of morphological features of these models which find a straightforward explanation by the invariant manifolds. Section 4 summarizes our conclusions.

\section{Model and invariant manifolds}

\subsection{Model}

The model of Kaufmann \& Contopoulos (1996) consists of a number of potential/density terms representing various components of a barred-spiral galaxy. In particular we have:

- a halo density term given by a Plummer sphere

$$
\rho_{\mathrm{h}}(r)=\frac{3 M_{\mathrm{h}}}{4 \pi b_{\mathrm{h}}^{3}}\left(1+\frac{r^{2}}{b_{\mathrm{h}}^{2}}\right)^{-5 / 2} \text {. }
$$

- A disk surface density given by an exponential law

$$
\Sigma_{\mathrm{d}}(r)=\Sigma_{0} \exp \left(-\epsilon_{\mathrm{d}} r\right)
$$

- A Ferrers bar with major axis aligned with the $y$-axis

$$
\rho_{\mathrm{b}}(x, y, z)=\frac{105 M_{\mathrm{b}}}{32 \pi a b c}\left(1-\frac{y^{2}}{a^{2}}-\frac{x^{2}}{b^{2}}-\frac{z^{2}}{c^{2}}\right)^{2}, \quad a>b>c,
$$

with

$$
1-\frac{y^{2}}{a^{2}}-\frac{x^{2}}{b^{2}}-\frac{z^{2}}{c^{2}} \geq 0
$$

- and a spiral perturbation in the potential

$$
V_{\mathrm{s}}(r, \theta)=A(r) r \exp \left(-\epsilon_{\mathrm{s}} r\right) \cos 2 \Phi
$$

where

$$
\begin{array}{ll}
\Phi=\frac{\ln (r / a)}{\tan i}-\theta & \text { if } r \geq a \\
\Phi=\theta & \text { if } r<a
\end{array}
$$

and

$$
A(r)=\left(\frac{A-A_{r}}{4}\right)\left(1+\tanh \left[\kappa_{1}\left(r-r_{1}\right)\right]\right)\left(1+\tanh \left[\kappa_{2}\left(r_{2}-r\right)\right]\right)+A_{r}
$$

The latter formula allows for a nearly constant amplitude $A(r)$ between the inner and outer cut-off radii $r_{1}$ and $r_{2}$, while the amplitude falls to a small value $A_{r}$ beyond $r_{2}$ or below $r_{1}$. We have in fact slightly modified Eq. (8) so that the potential becomes a smooth function of $\theta$ at $r=a$. Namely, we substitute (8) by the expression:

$\Phi=\frac{1}{2}[1+\tanh (2(r-a))] \frac{\ln (r / a)}{\tan i}-\theta$.

\begin{tabular}{|c|c|c|c|c|c|c|c|c|}
\hline \multicolumn{9}{|c|}{ Model A } \\
\hline Spiral: & $\begin{array}{c}\mathrm{A} \\
2000\end{array}$ & $\begin{array}{c}\varepsilon_{\mathrm{s}} \\
0.4\end{array}$ & $\begin{array}{c}i_{0} \\
-10^{\circ}\end{array}$ & $\begin{array}{l}r_{1} \\
1.5\end{array}$ & $\begin{array}{c}r_{2} \\
10.6\end{array}$ & $\begin{array}{c}\kappa_{1} \\
1\end{array}$ & $\begin{array}{c}\kappa_{2} \\
1\end{array}$ & $\begin{array}{c}\Delta \\
0.1\end{array}$ \\
\hline Bar: & $\begin{array}{c}M_{\mathrm{b}} \\
1.5\end{array}$ & $\begin{array}{c}a \\
5.5\end{array}$ & $\begin{array}{c}b \\
2.1\end{array}$ & $\begin{array}{c}c \\
0.55\end{array}$ & $\begin{array}{c}\Omega_{\mathrm{p}} \\
43.6\end{array}$ & & & \\
\hline Disk: & $\begin{array}{c}\Sigma_{0} \\
750\end{array}$ & $\begin{array}{c}\varepsilon_{\mathrm{d}} \\
0.235\end{array}$ & & & & & & \\
\hline Halo: & $\begin{array}{c}M_{\mathrm{h}} \\
27.5 \\
\end{array}$ & $\begin{array}{l}b_{\mathrm{h}} \\
12 \\
\end{array}$ & & & & & & \\
\hline \multicolumn{9}{|c|}{ Model B } \\
\hline Spiral: & $\begin{array}{c}\text { A } \\
9000\end{array}$ & $\begin{array}{c}\varepsilon_{\mathrm{S}} \\
1.2\end{array}$ & $\begin{array}{c}i_{0} \\
-10^{\circ}\end{array}$ & $\begin{array}{c}r_{1} \\
3\end{array}$ & $\begin{array}{c}r_{2} \\
5.6\end{array}$ & $\begin{array}{c}K_{1} \\
0.5\end{array}$ & $\begin{array}{c}K_{2} \\
0.5\end{array}$ & $\begin{array}{c}\Delta \\
0.1\end{array}$ \\
\hline Bar: & $\begin{array}{c}M_{\mathrm{b}} \\
0.18\end{array}$ & $\begin{array}{l}a \\
3\end{array}$ & $\begin{array}{c}b \\
0.4\end{array}$ & $\begin{array}{c}c \\
0.3\end{array}$ & $\begin{array}{c}\Omega_{\mathrm{p}} \\
32.2\end{array}$ & & & \\
\hline Disk: & $\begin{array}{c}\Sigma_{0} \\
250\end{array}$ & $\begin{array}{c}\varepsilon_{\mathrm{d}} \\
0.305\end{array}$ & & & & & & \\
\hline Halo: & $\begin{array}{l}M_{\mathrm{h}} \\
1.0\end{array}$ & $\begin{array}{c}b_{\mathrm{h}} \\
9 \\
\end{array}$ & & & & & & \\
\hline \multicolumn{9}{|c|}{ Model C } \\
\hline Spiral: & $\begin{array}{c}\mathrm{A} \\
3000\end{array}$ & $\begin{array}{c}\varepsilon_{\mathrm{s}} \\
0.4\end{array}$ & $\begin{array}{c}i_{0} \\
-6^{\circ}\end{array}$ & $\begin{array}{c}r_{1} \\
1\end{array}$ & $\begin{array}{l}r_{2} \\
10\end{array}$ & $\begin{array}{c}\kappa_{1} \\
0.5\end{array}$ & $\begin{array}{c}K_{2} \\
0.5\end{array}$ & $\begin{array}{c}\Delta \\
0.1\end{array}$ \\
\hline Bar: & $\begin{array}{c}M_{\mathrm{b}} \\
1.2\end{array}$ & $\begin{array}{c}a \\
4.8\end{array}$ & $\begin{array}{c}b \\
1.6\end{array}$ & $\begin{array}{c}c \\
0.48\end{array}$ & $\begin{array}{c}\Omega_{\mathrm{p}} \\
54.2\end{array}$ & & & \\
\hline Disk: & $\begin{array}{c}\Sigma_{0} \\
1475\end{array}$ & $\begin{array}{c}\varepsilon_{\mathrm{d}} \\
0.185\end{array}$ & & & & & & \\
\hline Halo: & $\begin{array}{c}M_{\mathrm{h}} \\
60.0\end{array}$ & $\begin{array}{l}b_{\mathrm{h}} \\
35\end{array}$ & & & & & & \\
\hline
\end{tabular}

Table 1. Parameters of models A, B, C (from Kaufmann \& Contopoulos 1996). The units are $\mathrm{km}^{2} \mathrm{~s}^{-2} \mathrm{kpc}^{-1}$ for $A, \mathrm{kpc}^{-1}$ for $\varepsilon_{\mathrm{s}}, \varepsilon_{\mathrm{d}}, \kappa_{1}$ and $\kappa_{2}, \mathrm{kpc}$ for $r_{1}, r_{2}, \Delta, a, b, c$, and $b_{\mathrm{h}}, 10^{10} M_{\odot}$ for $M_{\mathrm{h}}, M_{\mathrm{b}}, \mathrm{km} \mathrm{s}^{-1} \mathrm{kpc}^{-1}$ for $\Omega_{\mathrm{p}}$, and $M_{\odot} / \mathrm{pc}^{2}$ for $\Sigma_{0}$. Model $\mathrm{A}^{\prime}$ has the same parameters as model $\mathrm{A}$, except for the spiral amplitude $A=1000$ and the pitch angle $i_{0}=-9^{\circ}$. Model $\mathrm{B}^{\prime}$ has the same parameters as model B except for $A=2500$, $M_{\mathrm{b}}=0.09, i_{0}=-8^{\circ}, \Omega_{\mathrm{p}}=30.5 \mathrm{~km} \mathrm{~s}^{-1} \mathrm{kpc}^{-1}$.

The latter expression introduces a smoothing of the potential yielding a difference with respect to Eq. (8) which is $10 \%$ at the distance $r=a \pm 0.5 \mathrm{kpc}$ and only $2 \%$ at $r=a \pm 1 \mathrm{kpc}$.

A model is specified by a set of values for the parameters $M_{\mathrm{h}}$, $b_{\mathrm{h}}, \Sigma_{0}, \epsilon_{\mathrm{d}}, M_{\mathrm{b}}, a, b, c, \epsilon_{\mathrm{s}}, i, A, A_{r}, \kappa_{1}, r_{1}, \kappa_{2}$, and $r_{2}$, as well as the value of the pattern angular speed $\Omega_{\mathrm{p}}$. In Kaufmann $\&$ Contopoulos (1996), the parameters were adjusted so as to produce three different self-consistent models which present some features of three real barred galaxies. The criterion for selfconsistency was that the "response density", i.e., the density obtained by the superposition of many orbits in the fixed potential should match as closely as possible the imposed density represented by the above equations. The matching refers to a) the amplitudes of the surface density map on the disk plane, and b) the phases of the maxima of the bar and of the spiral arms, in the imposed and in the response models.

The parameters for the three models are given in Table 1. In the sequel we refer to these as model $\mathrm{A}, \mathrm{B}$, and $\mathrm{C}$. The value of $A_{r}$ for all three models, as well as the values of $r_{2}$ and $\kappa_{2}$ for model C are missing from Kaufmann \& Contopoulos (1996), where, however, it is noted that any (small) value of $A_{r}$, or of $r_{1}, r_{2}$ and $\kappa_{1}, \kappa_{2}$ does not influence the self-consistency (provided that $r_{2}$ is at the end of the spiral arms). For consistency with the remaining models, we have set $\kappa_{2}=\kappa_{1}$ and $r_{2}=10 \mathrm{kpc}$ in the case of model C, and $A_{r}=0$ in all three models.

Models A, B, C present some features of the galaxies NGC 3992, NGC 1073, and NGC 1398 respectively. As discussed below, the bar-spiral strengths induced by the parameters of model $\mathrm{C}$ are quite untypical of barred-spiral galaxies, 
(a)

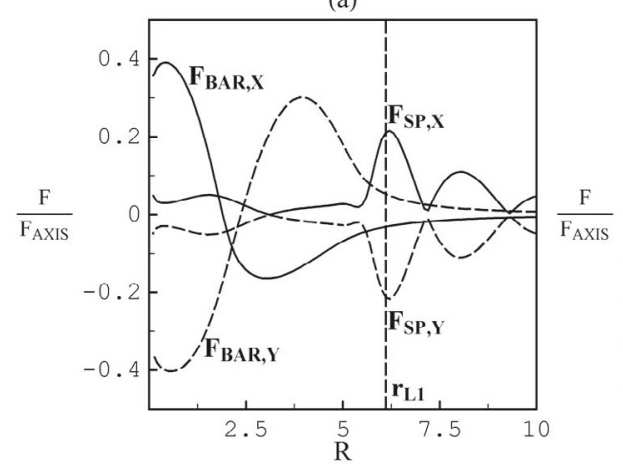

(b)

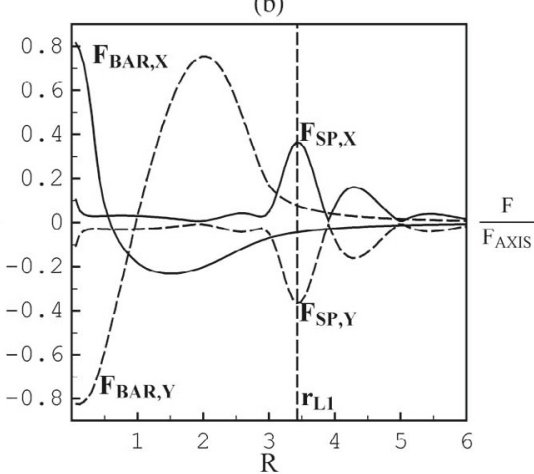

(c)

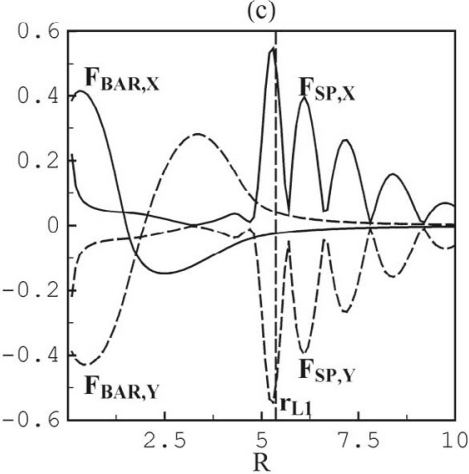

Fig. 2. The ratio of non-axisymmetric forces, due to the bar or to the spiral arms, versus total axisymmetric force, as a function of the distance $R$ along the $x$-axis (solid) or $y$-axis (dashed) for models A, B, and C (panels a), b), and c) respectively). The vertical dashed lines mark the distance of the $L_{1}$ or $L_{2}$ points in each case.

although still in the range allowed by observations. Thus, while the theory of the invariant manifolds worked well in all three models, we discuss in detail models $\mathrm{A}$ and $\mathrm{B}$, and only some exceptional features of model $\mathrm{C}$ interesting for dynamics. It should be pointed out that, while the choice of model parameters was partly based on observations (see Sects. 2, 3 of Kaufmann \& Contopoulos 1996), the so-obtained models are only rough representations of the referenced galaxies. For example, images of the galaxy NGC 3992 (e.g. in the I band, Tully et al. 1996) indicate the presence of at least one more arm of amplitude comparable to the main bi-symmetric pattern. Images of the galaxy NGC 1398, (e.g. in the $R$-band, Hammed \& Devereux 1999) reveal the existence of an inner ring structure which has no clearcut separation from the main spiral structure. Such morphological features are not captured by the potential/density model given by Eqs. (4)-(10). Finally, the use of a $n=2$ Ferrers bar model implies a steep drop of the bar force beyond the bar's limit which would be smoother in a $n=0$ or $n=1$ model, and it does also not account for a rectangular-like outline that is observed in many real bars.

These facts notwithstanding, the choice of potential parameters and pattern speeds as in Table 1 ensures the existence of a self-consistent solution for the response density, a fact which would by no means be implied in an arbitrary choice of potential model. Although we do not make explicit use of the library of orbits of the final solution in the present paper, and also no guarantee for the stability of the models is provided in Kaufmann \& Contopoulos (1996), the self-consistency property suggests that the spiral arms found in these galaxy models can be stellar dynamically supported. This conclusion is independent of the theory of the invariant manifolds, thus the latter theory can be tested against this conclusion.

The relative importance of the various non-axisymmetric components of the force with respect to the axisymmetric force vary with the distance from the center, as can be inferred from Fig. 2. The bar contributes to the forcing by both an axisymmetric and a non-axisymmetric component. The axisymmetric component is found as the azimuthally averaged radial bar force

$\bar{F}_{\mathrm{bar}, r}(r)=\frac{1}{2 \pi} \int_{0}^{2 \pi} F_{\mathrm{bar}, r}(r, \theta) \mathrm{d} \theta$,

where $F_{\mathrm{bar}, r}=\left(F_{\mathrm{bar}, x} x+F_{\mathrm{bar}, y} y\right) / r$. The total axisymmetric force is $F_{\mathrm{ax}}(r)=F_{\mathrm{disk}}(r)+F_{\text {halo }}(r)+\bar{F}_{\mathrm{bar}, r}(r)$. The non-axisymmetric bar force at a position $r, \theta$ is the difference $\boldsymbol{F}_{\text {non-ax }}=\boldsymbol{F}_{\text {bar }}-$ $\bar{F}_{\mathrm{bar}, r} \hat{\boldsymbol{e}}_{r}$ where $\hat{\boldsymbol{e}}_{r}$ denotes the unit vector in the radial direction.
On the other hand the spiral force corresponding to the potential $V_{\mathrm{s}}$ can be all considered as non-axisymmetric since the potential $V_{\mathrm{s}}$ only has a $\cos 2 \Phi$ dependent term. Figure 2 shows the absolute ratios $F_{\text {bar,non-ax }} / F_{\text {ax }}$ and $F_{\text {spiral }} / F_{\text {ax }}$ as a function of the radial distance $r$, along two directions, i.e., along the bar's major (dashed curves) and minor (solid curves) axes, for all three models.

In model A (Fig. 2a) the bar yields the dominant nonaxisymmetric perturbation at all distances up to a zone around corotation (shown as a vertical dashed line at $r=6.11 \mathrm{kpc}=$ $\left.r_{L 1}\right)$. The maximum amplitude of the non-axisymmetric bar force is 0.32 , corresponding to a peak of the $F_{\mathrm{bar}, y}$ curve at $R \simeq 4 \mathrm{kpc}$ (in all the panels of Fig. 2 the innermost local maxima or minima of the curves $F_{\mathrm{bar}, x}, F_{\mathrm{bar}, y}$ at $R \leq 1 \mathrm{kpc}$ are artificial, due to the weakening of the axisymmetric forces which, for a Plummer sphere, are exactly equal to zero at $R=0$ ). The inner width of the zone is found by the point where $F_{\text {bar,non-ax }}=F_{\text {spiral }}$, which is at a distance $r \simeq 5.75 \mathrm{kpc}$. Beyond that distance, the spiral term dominates over the bar term, reaching a maximum amplitude equal to 0.21 with respect to the axisymmetric background. The oscillations of the spiral force beyond corotation are due to the logarithmic dependence of the argument $\Phi$ in (7) on $r$, a fact causing successive maxima and minima of the spiral force at successive periods of length $2 \pi$ of the argument $\Phi$. The first maximum, around corotation, is the most important. The width of the oscillation from this maximum to the next defines an approximate value of the radial wavelength of the spiral density wave, which is $\Delta r \simeq 1 \mathrm{kpc}$.

In model B (Fig. 2b) the maximum amplitude of the nonaxisymmetric bar force reaches the value 0.75 (for $F_{\mathrm{bar}, y}$ at $R \simeq 2 \mathrm{kpc}$ ), implying that the bar is quite strong inside corotation. Nevertheless, even in this galaxy the spiral force becomes dominant over the bar's non-axisymmetric perturbation around and beyond corotation. The zone around the first maximum of the spiral force defines a radial wavelength of the spiral density wave $\Delta r \simeq 1 \mathrm{kpc}$. The first peak of the spiral force is again found to be at a distance very close to the corotation radius $r=r_{L 1}=3.43$ and the amplitude of this peak is 0.35 .

Finally, in model C (Fig. 2c) the spiral perturbation near and beyond corotation reaches such a high amplitude (maximum $=$ $0.57)$, that it becomes even stronger than the maximum amplitude of the bar's perturbation $\left(\simeq 0.3\right.$ for $F_{\text {bar, } y}$ at $\left.R \simeq 3.5 \mathrm{kpc}\right)$ which takes place well inside corotation. Furthermore, the spiral arms are tightly wound (the radial wavelength is estimated as $\Delta r \simeq 0.5 \mathrm{kpc}$ ), and the spiral arms extend to cover about one azimuthal period $2 \pi$. Thus, model $\mathrm{C}$ is exceptional and will not 


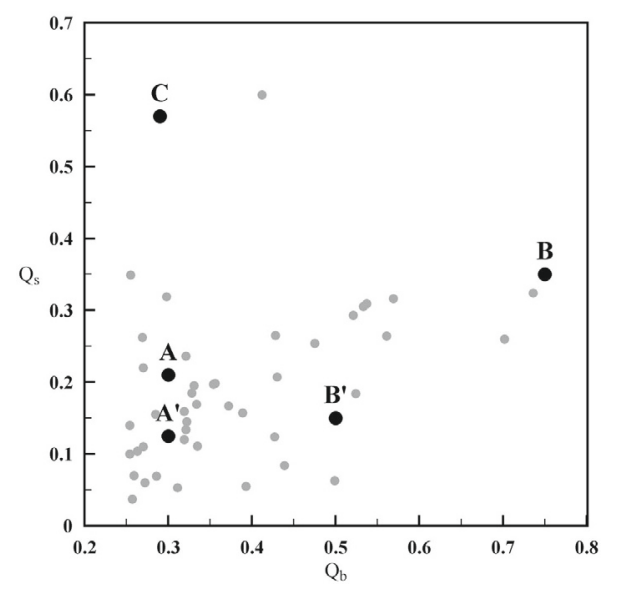

Fig. 3. The $Q_{\text {s }}$ vs. $Q_{\mathrm{b}}$ values in observed $\mathrm{SB}$ galaxies (gray points from the Buta et al. (2005) sample) and our models A, A', B, B', C (black dots).

be discussed in detail in the sequel. Only a feature of this model interesting for dynamics is discussed in Sect. 2.3.

The bar-spiral amplitudes of models A and B define strongly nonlinear models, which are above the average but inside the range of bar-spiral strengths found by recent observations (Laurikainen \& Salo 2002; Buta et al. 2005). In the latter works the maximum value of the ratio of the tangential force versus the radial force for the bar and spiral components is denoted by $Q_{\mathrm{b}}$ and $Q_{\mathrm{s}}$ respectively. The average values for SB galaxies in the sample of Buta et al. (2005) are $\left\langle Q_{\mathrm{b}}\right\rangle=0.29$ and $\left\langle Q_{\mathrm{s}}\right\rangle=0.17$. Model A yields $Q_{\mathrm{b}}=0.3$ and $Q_{\mathrm{s}}=0.21$. Model B yields $Q_{\mathrm{b}}=0.75$ and $Q_{\mathrm{s}}=0.35$, both values being a factor 1.3 larger than the specific estimates reported by Buta et al. (2005) for the galaxy NGC $1073\left(Q_{\mathrm{b}}=0.56, Q_{\mathrm{s}}=0.26\right)$, to which model $\mathrm{B}$ is associated. In order to have a more representative sample of models in which the theory of the invariant manifolds is to be tested, two "weak" models, $\mathrm{A}^{\prime}$ and $\mathrm{B}^{\prime}$, are also considered, which were created by varying some parameters of models $\mathrm{A}$ and $\mathrm{B}$. In model $\mathrm{A}^{\prime}$ the spiral amplitude is $A=1000$, i.e. half the value of model A (see Table 1). In model B' the bar mass is $M_{\mathrm{b}}=0.09$ and the spiral amplitude $A=2500$. Since these changes are rather arbitrary, there is no guarantee of selfconsistency of the new models. However, a rough criterion of self-consistency (Sect. 2.2) can be established if the pitch angle is also slightly varied in both models $\left(i_{0}=-9^{0}\right.$ in model $\mathrm{A}^{\prime}$ and $i_{0}=-8^{0}$ in model $\left.\mathrm{B}^{\prime}\right)$. The resulting $Q_{\mathrm{b}}$ and $Q_{\mathrm{s}}$ values are $Q_{\mathrm{b}}=0.3, Q_{\mathrm{s}}=0.125$ for model $\mathrm{A}^{\prime}$ and $Q_{\mathrm{b}}=0.5, Q_{\mathrm{s}}=0.15$ for model $\mathrm{B}^{\prime}$. The spiral strengths of models $\mathrm{A}^{\prime}, \mathrm{B}^{\prime}$ are well below the average of the Buta et al. sample for SB galaxies. Thus, the mean values $\left\langle Q_{\mathrm{b}}\right\rangle$ and $\left\langle Q_{\mathrm{s}}\right\rangle$ of the four models $\mathrm{A}, \mathrm{B}, \mathrm{A}^{\prime}, \mathrm{B}^{\prime}$ become both representative of the average values found in the observations. On the other hand, the value $Q_{\mathrm{s}}=0.57$ of model C is untypical although still in the range of the observations (Fig. 3).

\subsection{Phase portraits and invariant manifolds}

The first result of the analysis of the invariant manifolds can now be demonstrated with the help of Figs. 4 to 6. Figure 4a shows the phase portrait (surface of section $\left(\theta, p_{\theta}\right)$ corresponding to the apocentric positions $\dot{r}=0, \dot{p}_{r}<0$ ) in the case of model A, for a value of the Jacobi constant $E_{\mathrm{J}}=-1.91 \times 10^{5}$, which is close to the value $E_{\mathrm{J}, L 1}=-1.915 \times 10^{5}$. Figure $4 \mathrm{c}$ shows the same portrait for $E_{\mathrm{J}}=-1.91 \times 10^{5}$ in a so called "aligned spiral" version of model A in which the angle $\Phi$ in Eq. (10) is replaced by $\theta$ throughout the whole radial extent of the spiral arms. This means to artificially "align" the spiral arms as extensions of the bar along the latter's major axis. By this way we measure the effect of only increasing the amplitude of the non-axisymmetric perturbation on the form of the invariant manifolds, while in the original model the manifolds are affected both by the strength of the non-axisymmetric perturbation and by the azimuthal displacement of the unstable Lagrangian points with respect to the bar's major axis.

In Figs. 4a,c the points marked PL1, PL2 correspond to the fixed points of the PL1 or PL2 short-period orbits which are close to the positions of the unstable equilibria $L_{1}, L_{2}$. Furthermore, the thick dots show the intersection of the unstable manifolds $\mathcal{W}_{\mathrm{PL} 1}^{U}$ and $\mathcal{W}_{\mathrm{PL} 2}^{U}$ with the surface of section. In order to facilitate the reading of these diagrams, we note that, for $p_{r}=0$ (apsides), beyond some radius $r_{0} \simeq 1 \mathrm{kpc}$, Eq. (1) yields that $r$ increases nearly monotonically with $p_{\theta}$ in all azimuthal directions of a fixed angle $\theta$ (a small reversal of this monotonic relation, due to the non-axisymmetric potential terms, is only observed at angles $\theta \simeq \pi / 2$ and in a small interval of radii, of width $\Delta r<0.1 \mathrm{kpc}$ around $r=5 \mathrm{kpc}$; the monotonic relation is re-established after crossing this interval). Thus, in Figs. 4a,c the semi-plane of the phase portrait with $p_{\theta}>p_{\theta, L 1}$ means apocentric positions outside corotation, while $p_{\theta}<p_{\theta, L 1}$ means apocentric positions inside corotation. Note that in this and in all subsequent plots of phase portraits the values of $p_{\theta}$ are normalized with respect to the value $a^{2} \Omega_{\mathrm{p}}$, corresponding to the angular momentum in the rest frame of a circular orbit at a radius $r=a$ under the action of only the axisymmetric potential.

The main remarks about the comparison of the two phase portraits are now the following:

- in both portraits chaos is pronounced inside corotation (for $\left.p_{\theta}<p_{\theta, L 1}\right)$, and the domain of inner invariant KAM curves is deeply inside the bar (at values of $p_{\theta}$ about or below 0.25 ). Such extended chaotic domains are responsible for the termination of the bar;

- outside corotation (for $p_{\theta}>p_{\theta, L 1}$ ), a layer of outer KAM curves has been destroyed in both portraits. This is caused mainly by the growth of the chaotic layer around the unstable $-6 / 1$ periodic orbit, which produces a resonance overlap with the chaotic layer of the PL1,2 unstable periodic orbit (negative signs indicate a resonance outside corotation, for which the motion is retrograde in the azimuthal direction). As a result, the chaotic domain extends up to values of $p_{\theta} \simeq 1.5$, and the first rotational KAM curves appear a little inside the $-4 / 1$ resonance. The domain around the outer Lindblad resonance is almost entirely filled either by rotational KAM curves or by "resonant" curves around the $-2: 1$ stable periodic orbits. The islands of stability of the $-2: 1$ resonance have a larger width in the "aligned spiral" model (Fig. 4c) because by aligning the spiral perturbation the amplitude of the total non-axisymmetric perturbation increases effectively at large distances from corotation (the width of resonances scales as a power-law of the non-axisymmetric perturbation (see e.g. Contopoulos 2002);

- the white circular domains devoid of points, embedded in the chaotic sea of both portraits, correspond to prohibited domains of motion, for $\dot{r}=0$ and for the selected values of the Jacobi constant. Such domains exist when $E_{\mathrm{J}}<E_{\mathrm{J}, L 4}$ (equal to $E_{\mathrm{J}, L 4}=-1.873 \times 10^{5}$ );

- the most important difference between the two portraits is that in the case of the true spiral term turned on (Fig. 4a) the prohibited domains lose their azimuthal symmetry with respect 

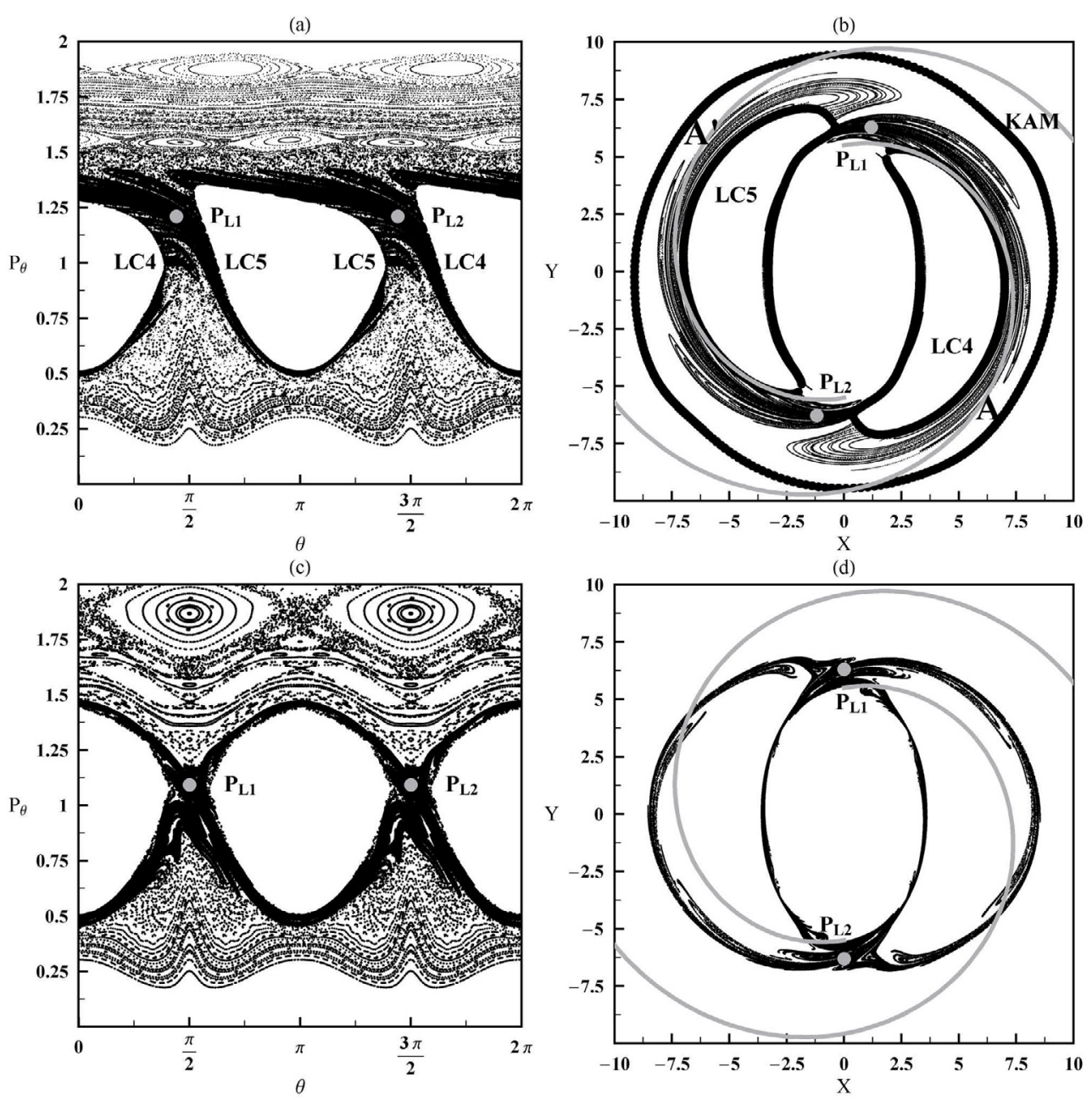

Fig. 4. a) Phase portrait near corotation $\left(E_{\mathrm{J}}=-1.911 \times 10^{5}\right)$ in the case of model A. The thick gray points mark the position of the fixed points of the PL1 and PL2 orbits. The thick dark lines are the invariant manifolds $\mathcal{W}_{\mathrm{PL} 1}^{U}$ and $\mathcal{W}_{\mathrm{PL} 2}^{U}$. b) Projection of the manifolds of a) in the configuration space. The gray spiral curves correspond to the density maxima of the imposed spiral arms of the full model. The curves marked LC4, LC5 and KAM are commented in the text. c), d) Same as a), b) in the "aligned spiral" $(\Phi=\theta)$ version of model A, in which the minima of the spiral potential term are aligned with the bar, and $E_{\mathrm{J}}=-1.91 \times 10^{5}$.

to the values $\theta=0$ (equal to $2 \pi(\bmod 2 \pi))$, or $\theta=\pi$ referring to the positions of the stable Lagrangian points $L_{4}$ and $L_{5}$. Such a symmetry is perfect in the aligned spiral case (Fig. 4c). The limiting boundaries of the prohibited domains are denoted by "LC4", "LC5" in Fig. 4. The azimuthal deformation of the prohibited domains corresponds to an azimuthal deformation of the associated banana-like prohibited domains appearing in the configuration space (i.e. the disk plane). These domains are similar but should not be confused with the domains delimited by the zero velocity curves of the effective potential in the rotating frame, i.e. $V_{\text {eff }}=V(r, \theta)-\Omega_{\mathrm{p}}^{2} r^{2} / 2$.

It turns out that the azimuthal deformation of the limiting boundaries LC4 and LC5 is a crucial difference related to the morphology of the spiral arms. In both Figs. 4a,c the manifolds are well developed inside and outside corotation (below and above PL1). In particular, the invariant manifolds form conspicuous lobes and foldings which are typical of systems having a large degree of the so-called homoclinic chaos. However, when the manifolds are plotted in the configuration space (Figs. 4b,d), the azimuthal deformation of the limiting boundaries LC4 and LC5 in the truly spiral model (Fig. 4b) causes the manifolds to be also azimuthally deformed in a way so as to closely support the imposed spiral arms up to an azimuth $\theta^{\prime} \approx 3 \pi / 4$ measured clockwise from either $L_{1}$ or $L_{2} \mathrm{~s}$. The end of this agreement is in two regions (marked $\mathrm{A}$ and $\mathrm{A}^{\prime}$ ) in which the manifolds $W_{\mathrm{PL} 1}^{U}$ and $\mathcal{W}_{\mathrm{PL} 2}^{U}$ lose contact from the locus of maxima predicted by Eq. (8). Beyond this distance, the manifolds exhibit a typical behavior called a "bridge" in our previous works (Voglis et al. 2006a; Tsoutsis et al. 2008). Namely, the manifold $\mathcal{W}_{\text {PL1 }}^{U}$ forms an inner spur consisting of a number of lobes connecting segments of the manifold from A to their continuation, which starts in the neighborhood of the other periodic orbit, PL2. From that point on the oscillations of the manifold $\mathcal{W}_{\mathrm{PL} 1}^{U}$ start supporting both the spiral arm emanating from $L_{2}$ and the chaotic layer marking the border of the bar. When the manifold is calculated for even longer lengths, we find that the higher order lobes of the manifold from $L_{1}$, after reaching the neighborhood of $L_{2}$, come again close to $L_{1}$ in directions nearly parallel to those formed by the low order lobes of the same manifold. This causes an enhancement of the density close to $L_{1}$ along the direction of the imposed spiral arms, i.e., the manifolds support the self-consistency of the spiral arms.

On the other hand, the manifolds of the "aligned spiral" version of model A (Fig. 4d), calculated up to a length comparable to that of the manifolds of Fig. 4b, show no support of a spiral structure, but only yield a thick ring-like structure. The 

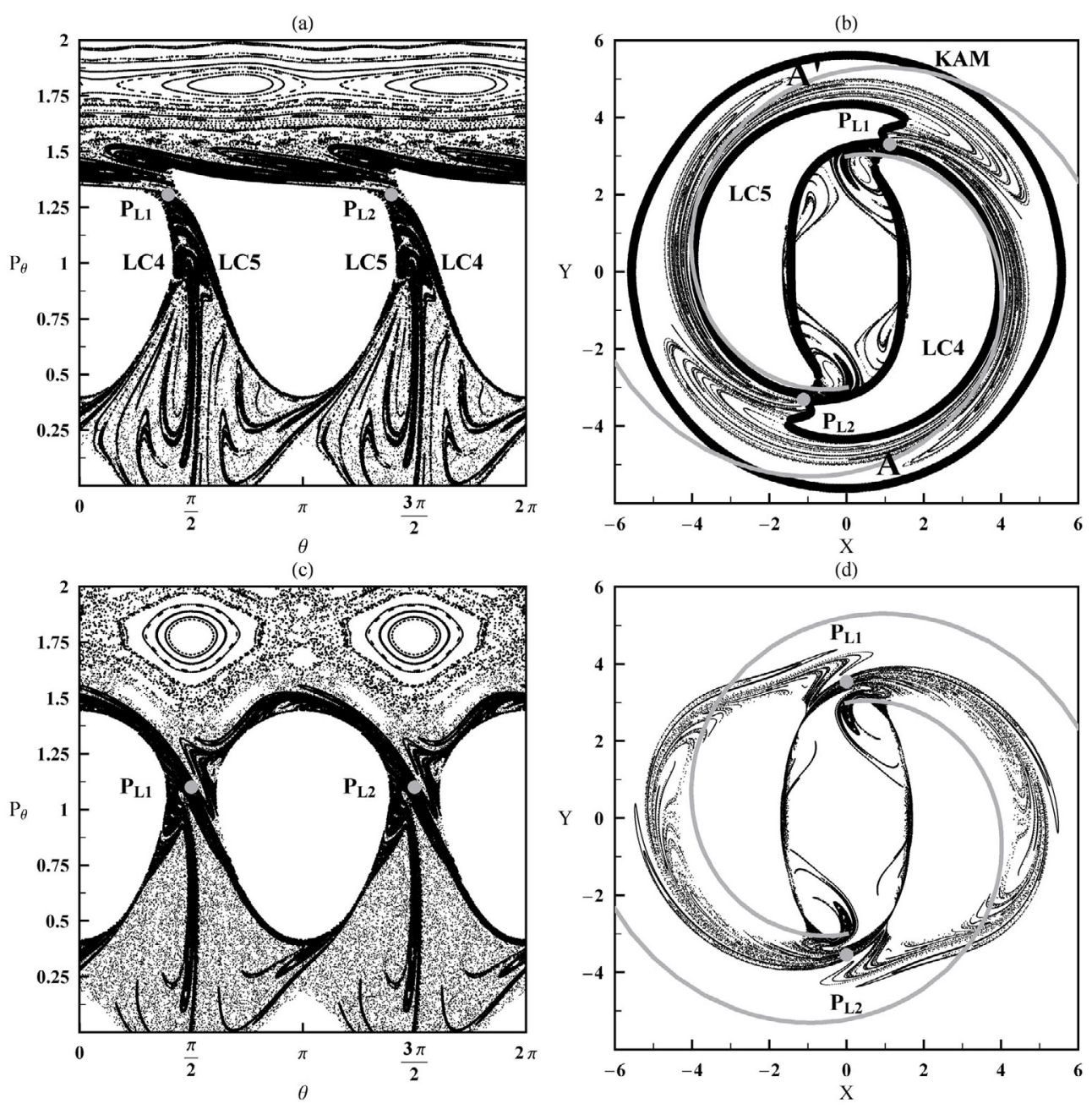

Fig. 5. Same as in Fig. 4, but for model B. The Jacobi constant is $E_{\mathrm{J}}=-2.98 \times 10^{4}$ in a) and b), and $E_{\mathrm{J}}=-2.97 \times 10^{4}$ in c), d).

thickness of the manifolds of Figs. $4 \mathrm{~b}, \mathrm{~d}$ is determined by the degree of chaos in Figs. 4a,c. The degree of chaos is determined by the amplitude of the non-axisymmetric perturbation. This is expected from dynamical systems theory, since the overlapping of resonances, which is the main source of production of chaos, depends on the width of the different resonant layers near corotation, which, in turn, depends on only the amplitude of the perturbation.

A more elaborate analysis (Sect. 2.3) shows that, while the outermost radial limit of the invariant manifolds is posed by the existence of absolute barriers, i.e. rotational KAM tori (marked "KAM" in Fig. 4b), more stringent limits are practically posed by partial barriers, i.e. cantori, which limit the diffusion within a chaotic zone. Provided these limits, the azimuthal deformation of the invariant manifolds is the crucial factor for the production by them of response spiral arms. This, in turn, is determined by the form of the limiting boundaries LC4 and LC5. The theoretical derivation of these boundaries is given in (Sect. 2.3).

Figure 5 shows the same phenomena in the case of model B. The qualitative resemblance between Figs. $4 a$, b and 5a,b is obvious, although the azimuthal deformation of the limiting boundaries LC4 and LC5 is more pronounced in Fig. 5b than in Fig. 4b. Also in this model the manifold exhibits a bridge starting at an angle $\theta^{\prime} \simeq 3 \pi / 4$ clockwise from $L_{1}$ or $L_{2}$ (points $A, A^{\prime}$ ), as well as inner spurs connecting segments of it along both spiral arms and along the border of the bar. Another feature of Fig. $5 \mathrm{~b}$ is that the inner branch of the invariant manifold (inside the bar) is developed in a domain occupying about one fourth of the total extent of the bar. This implies that a substantial part of the bar in the domain near corotation is supported by chaotic orbits. In fact the non-axisymmetric forcing in model B is much stronger inside corotation than in model $\mathrm{A}$, a fact causing the destruction of all the inner KAM curves down to $p_{\theta}=0$ (Fig. 5a). Such a type of chaos may lead to a number of observational consequences, photometric and kinematic, a list of which have been enumerated by Grosbøl (2003). Finally, the azimuthal deformation of the maxima of the spiral term with respect to the bar's major axis also turn out to be the crucial factor for the production by the manifolds of response spiral arms. In fact, by comparing Figs. 5a,b with the respective figures in the "aligned spiral" version of model B (Figs. 5c,d) we see that the manifolds in the latter case present some asymmetry as well as a large thickness, due to the high value of the non-axisymmetric perturbation, but they still largely deviate from the spiral pattern (gray locus), which was closely followed by the manifolds of the non-aligned model (Fig. 5b).

Figure 6 shows the phase portrait structure near corotation in the "weak spiral" models A' (Fig. 6a) and B' (Fig. 6b). The invariant manifolds $\mathcal{W}_{\mathrm{PL} 1}^{U}$ and $\mathcal{W}_{\mathrm{PL} 2}^{U}$ are also plotted, and the counterparts of these plots in the configuration space are shown in Figs. 6b and 6d respectively. As expected, in both models chaos is considerably reduced with respect to the strongly nonlinear models A, B, and it is only limited in a narrow zone in the corotation region. Further away, the phase space is filled by 
(a)
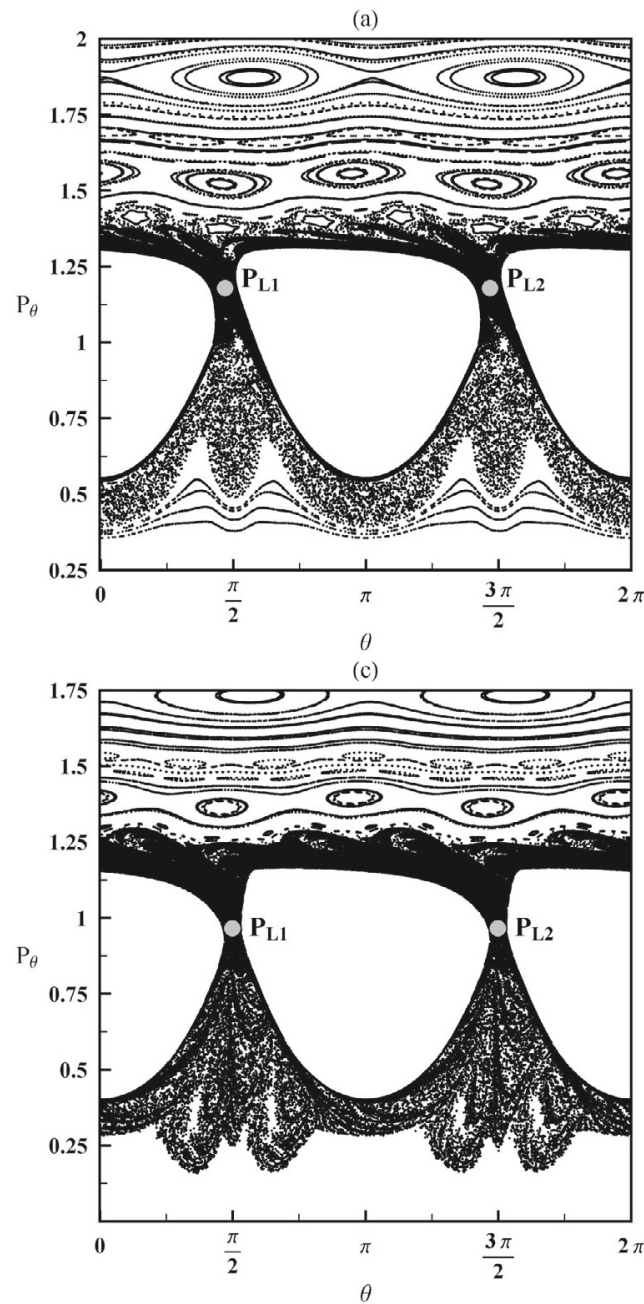

(b)

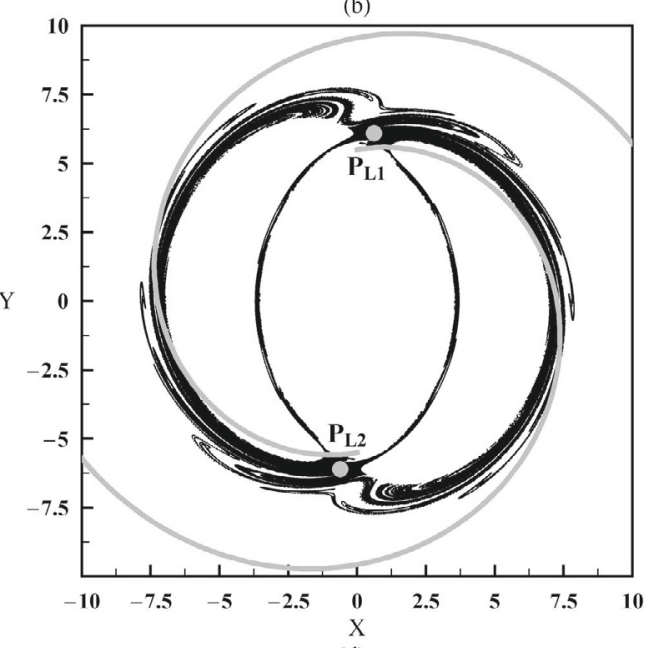

(d)

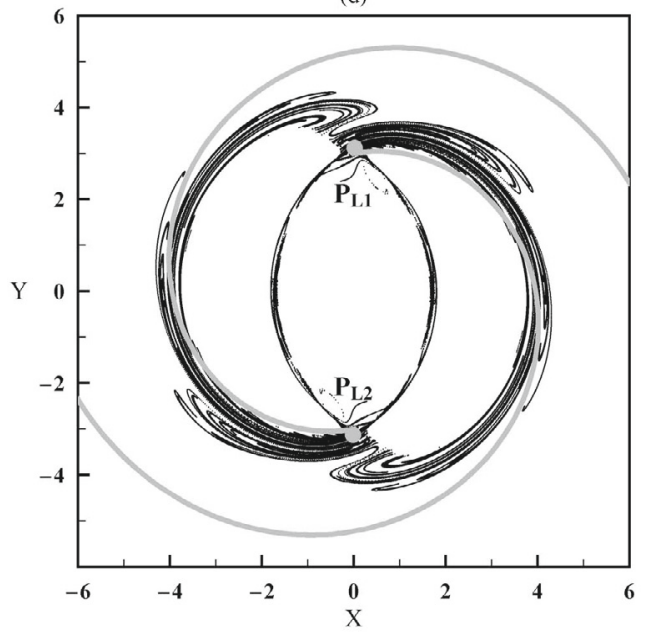

Fig. 6. a), b) Same as in Figs. $4 \mathrm{a}, \mathrm{b}$ but for the model $\mathrm{A}^{\prime}$, and $E_{\mathrm{J}}=-1.91 \times 10^{5}$. c), d) same as in Figs. $5 \mathrm{a}$,b but for the model $\mathrm{B}^{\prime}$ and $E_{\mathrm{J}}=-2.77 \times 10^{4}$.

invariant tori which occupy most of the phase space volume already at the $-4: 1$ resonance.

The $Q_{\mathrm{s}}$ value of model $\mathrm{A}^{\prime}$ is $Q_{\mathrm{s}}=0.125$, and this is its only difference with respect to model A, which has $Q_{\mathrm{s}}=0.21$. The thickness of the invariant manifolds is thus reduced with respect to the thickness of the manifolds of model A (compare Figs. 6b and $4 \mathrm{~b}$ ). However, the azimuthal deformation of the manifolds is still large enough to fit the locus of the imposed spiral arms up to an angle $\theta^{\prime}=\pi / 2$ clockwise from $L_{1}$ or $L_{2}$, i.e. the manifolds support quarter turn spiral arms. In the case of model B' (Fig. 6d) we have $Q_{\mathrm{s}}=0.15$, which is close but still below the average value $\left\langle Q_{\mathrm{s}}\right\rangle=0.17$ of the Buta et al. (2005) sample. At the value $Q_{\mathrm{s}}=0.15$ the region of homoclinic chaos formed by the lobes of the manifolds near $L_{1}$ or $L_{2}$ is already well developed, and the "inner spurs" are clearly distinguishable. In fact, in model B' a small adjustment of the pattern speed $\left(\Omega_{\mathrm{p}}=30.5\right.$ instead of $32.5 \mathrm{~km} \mathrm{~s}^{-1} \mathrm{kpc}^{-1}$, as was in model B) yielded the best fit of the invariant manifolds to the imposed spiral arms. Such a fit can now be considered as a rough criterion of self-consistency. We see that the formation of bridges in the manifolds of Fig. 6d result in that the higher order lobes of the manifold make oscillations which enhance the density along the manifolds' unstable directions all the way from $L_{1}$ or $L_{2}$. Thus the manifolds support, again, the imposed spiral arms in a self-consistent way. In fact, since the overall thickness of the domains covered by the invariant manifolds increases, the manifolds support the spiral structure up to an angle $\theta^{\prime}$ larger than $\pi / 2$, i.e. along a length larger than in model $\mathrm{A}^{\prime}$.

In conclusion, there is a clear morphological continuity of the structures produced by the invariant manifolds, from rings to quarter turn spirals, and then to fully developed spiral arms, as the value of $Q_{\mathrm{s}}$ increases. The azimuthal displacement of the unstable Lagrangian points is responsible for the manifolds producing a spiral-like response, while the increase of the amplitude $Q_{\mathrm{S}}$ extends the support of the spiral structure to higher angles $\theta^{\prime}$. Such a morphological continuity of the invariant manifolds is suggestive of it being a real morphological feature of barred galaxies.

\subsection{Theoretical modeling}

A first estimate of the azimuthal deformation of the limiting boundaries LC4 and LC5 can be done by calculating the azimuthal displacement of the unstable Lagrangian points $L_{1,2}$ when $V_{\text {spiral }}$ is turned on (Figs. 7a,b, schematic). This can be judged from the form of the equipotential curves (called hereafter the "curves of zero velocity", CZV) in the rotating frame, i.e., the level curves of

$V_{\mathrm{eff}}=V(r, \theta)-\Omega_{\mathrm{p}} r^{2} / 2=E_{\mathrm{J}}$. 

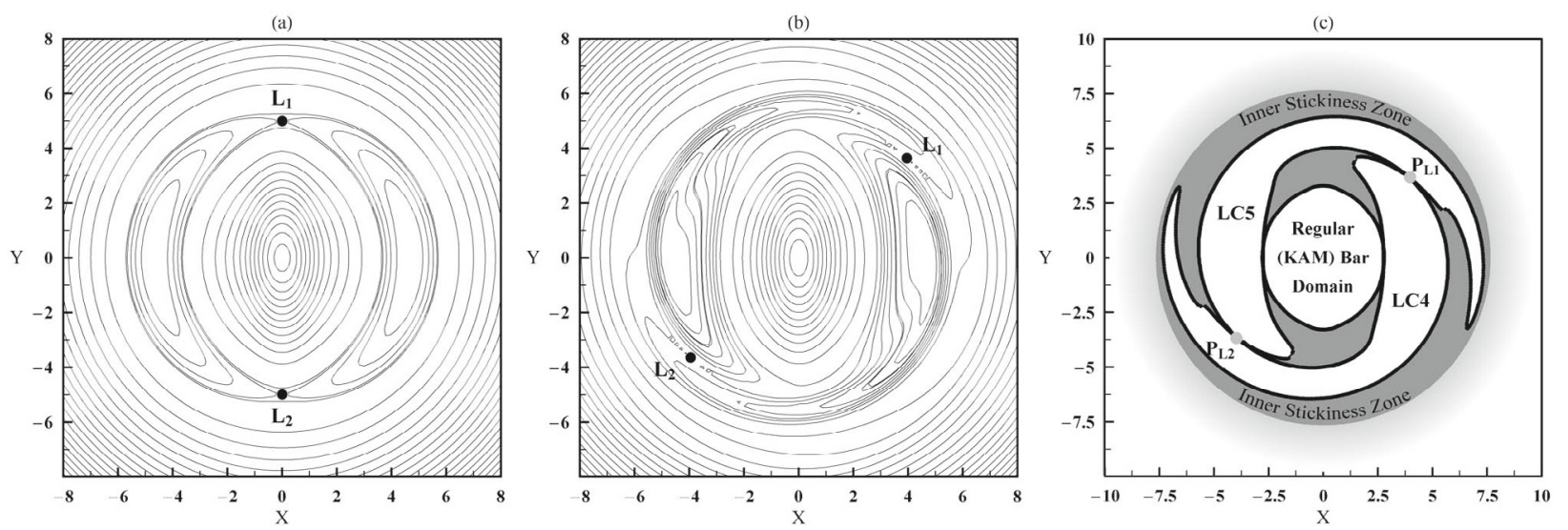

Fig. 7. The curves of zero velocity (equipotential curves of the effective potential $V_{\text {eff }}$ ) shown schematically when the non-axisymmetric perturbation is a) aligned to the bar, and b) has a spiral component. c) A schematic figure of the basic theory according to which the structure of the chaotic phase space in the corotation region supports of trailing spiral arms. The spiral arms are confined in an inner stickiness zone (dark) delimited by cantori which are the remnants of rotational KAM tori outside corotation. Inside the bar, the same chaotic zone is delimited by rotational KAM tori (regular bar domain). The chaotic zone between the curves LC4 and LC5 and the boundary of the stickiness zone has a spiral shape, due to the deformation of the limiting curves LC4 and LC5 caused by the azimuthal displacement of L1 and L2 with respect to the bar's major axis.

For a logarithmic spiral, the azimuthal shift of $L_{1}$ is given by

$\Delta \theta=\frac{1}{\tan i_{0}}\left|\ln \frac{r_{L_{1}}}{a}\right|$

where $\Delta \theta$ is taken clockwise from the bar's major axis. The azimuthal deformation of the CZVs takes place in a narrow zone around corotation (Fig. 7b), while, for $r$ small, the curves remain practically aligned to the bar. This is because the spiral potential term (7) (with $\Phi$ given by Eq. (10)) adds practically a $\cos 2 \theta$ contribution to the bar's term at radial distances $r<a$. The stable Lagrangian points $L_{4,5}$ are shifted counterclockwise with respect to the bar's minor axis, although by a smaller angle than that of the unstable points $L_{1}, L_{2}$.

The equipotential curves of $V_{\text {eff }}$ do not provide the strictest limit of allowed motions on the apocentric surface of section $\left(\theta, p_{\theta}\right)$, or $(\theta, r)$, for $p_{r}=0, \dot{p}_{r}<0$. The form of the limiting curves in the configuration space is shown schematically in Fig. 7c (curves LC4 and LC5, encircling the stable Lagrangian points $L_{4}, L_{5}$ ). These curves are derived by the requirement that, for any fixed angle $\theta$ and angular momentum $p_{\theta}$, the curve of the function

$\bar{V}\left(r ; \theta, p_{\theta}\right)=\frac{p_{\theta}^{2}}{2 r^{2}}-\Omega_{\mathrm{p}} p_{\theta}+V(r, \theta)$

be tangent to the line $\bar{V}=E_{\mathrm{J}}$ at the point where $\bar{V}=E_{\mathrm{J}}$ has a global minimum. The minimum is calculated by the root $r=$ $r_{\mathrm{m}}\left(\theta, p_{\theta}\right)$ of

$\frac{\partial \bar{V}}{\partial r}=-\frac{p_{\theta}^{2}}{r^{3}}+\frac{\partial V}{\partial r}=0$.

The limiting values of $p_{\theta}$, for fixed $\theta$, are then found via the roots for $p_{\theta}$ of

$\bar{V}\left(r_{\mathrm{m}}\left(\theta, p_{\theta}\right), \theta, p_{\theta}\right)=E_{\mathrm{J}}$.

The limiting curves LC4 and LC5 in the configuration space, derived from Eqs. (14) and (15) are outside the limiting curves provided by the CZVs defined through Eq. (12). This is due to the fact that these limits now refer to $\dot{r}=0$, while in the case of Eq. (12) the total velocity in the rotating frame is equal to zero (details are given in Appendix A). By virtue of these facts, the curves LC4 and LC5 exhibit also a spiral-like azimuthal deformation, which is even more pronounced than that of the CZVs.

The gray domain in Fig. 7c shows the permissible apocentric positions of the orbits under a fixed value of the Jacobi constant close to the corotation value. The interior gray domain between LC4 and LC5 roughly marks the extent of the bar. On the other hand, the positions of the PL1 and PL2 points are in very narrow strips of permissible apocentric positions separating the inner right part of the LC4 curve from the outer right part of the LC5 curve and vice versa. The invariant manifolds $W_{\mathrm{PL} 1,2}^{U}$ emanating from these points necessarily follow the narrow strips leading to the outer gray domain, thus they yield locally the form of spiral arms.

The evolution of the invariant manifolds further away from PL1 or PL2 is determined by the resonant structure in the outer corotation zone. The existence of many resonances accumulating in a narrow range of distances near the corotation radius causes a chaotic layer in this region, formed by the mechanism of resonance overlap. Figure 8 makes a zoom to the phase portraits of the models considered, in order to demonstrate the relevant phenomena. Figures $8 \mathrm{a}, \mathrm{b}$ are zooms to the phase portraits of Figs. 4a, and 5a, referring to the models A and B. In both cases we find a chaotic layer extending up to $p_{\theta} \simeq 1.5$, which is delimited by a rotational KAM torus at $p_{\theta}=1.5$ (marked $\mathrm{KAM}$ ). This torus is just below the $-4 / 1$ resonance, which is stable at the value of the Jacobi constant $E_{\mathrm{J}}=-1.911 \times 10^{5}$. On the other hand, most islands of stability of resonances $-m / 1$, with $m>4$, which are closer to corotation, have been destroyed. Their destruction is followed by the destruction of KAM tori with irrational rotation numbers, which, according to the standard theory, are transformed into cantori. Such cantori limit the chaotic flux through their gaps, and this fact causes some stickiness in a zone very close to the PL1 and PL2 fixed points. Stickiness phenomena of this type have been explicitly demonstrated and studied in simple models of the dynamical systems theory (see e.g. Efthymiopoulos et al. 1997; Contopoulos et al. 1999; Contopoulos \& Harsoula 2008), and they have also been observed in our $N$-Body simulations of barred-spiral galaxies (Tsoutsis et al. 2008). The main outcome of these studies is that the cantori in a large chaotic sea act as partial barriers slowing down considerably the rate of escape (or the diffusion) of the 
(a)

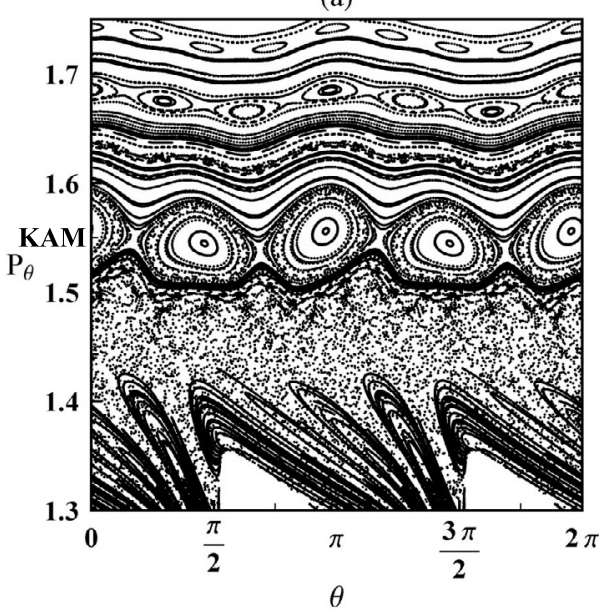

(c)

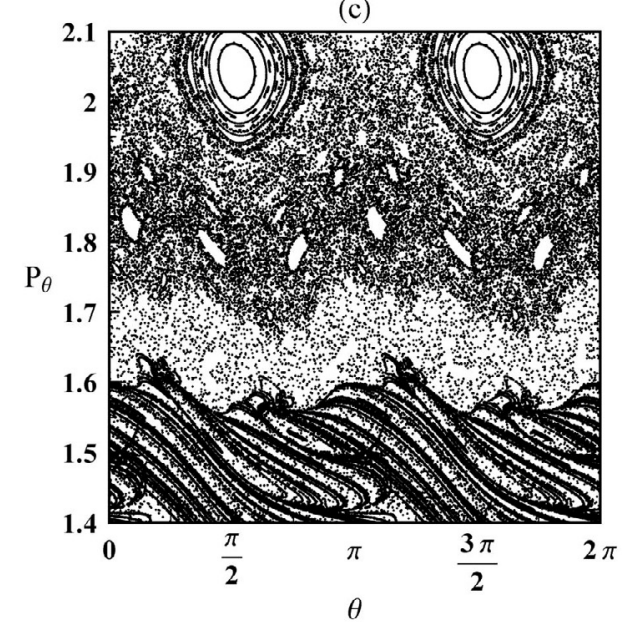

(b)

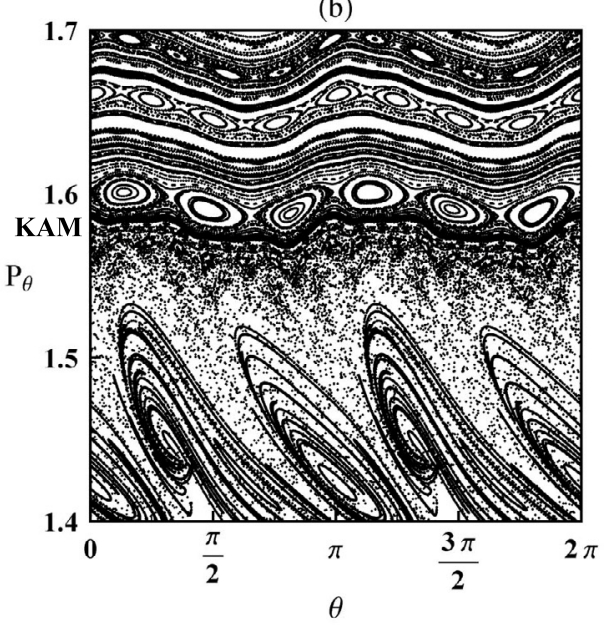

(d)

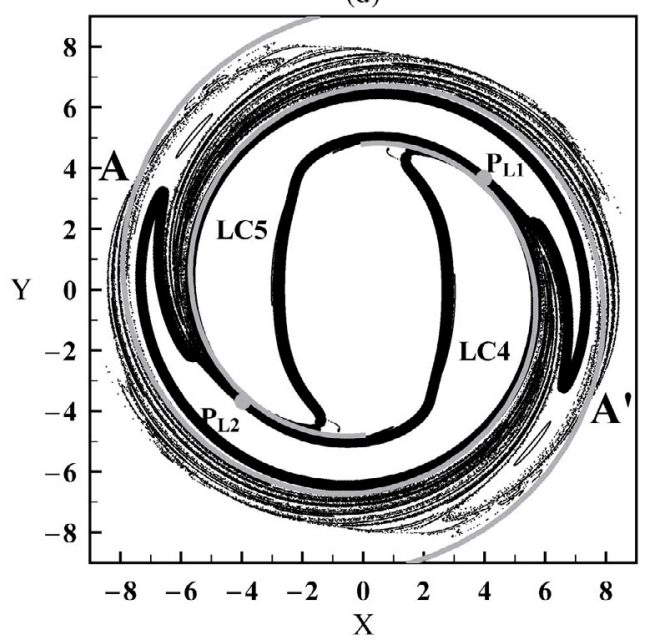

Fig. 8. The resonant phase space structure in the corotation region in the cases of a) model A with $E_{\mathrm{J}}=-1.911 \times 10^{5}$, b) model B with $E_{\mathrm{J}}=$ $-2.98 \times 10^{4}$, and c) model C with $E_{\mathrm{J}}=-2.912 \times 10^{5}$. The invariant manifolds of the PL1 and PL2 orbits are over-plotted (thick lines). The bold rotational KAM curves near $p_{\theta}=1.5$ in a) and $p_{\theta}=1.57$ in b) are very close to the inner boundary of the regular domain beyond corotation. In c) there is no such boundary at least up to the outer Lindblad resonance. The projection of the rotational curves of a) and b) marked KAM, as well as of the curves LC4 and LC5, in the configuration space, yields the respective outer closed curves in Figs. 4b, 5b. d) Same as in Fig. 4b but for the model C, and Jacobi constant $E_{\mathrm{J}}=-2.912 \times 10^{5}$.

chaotic orbits with initial conditions along or near an invariant manifold.

In the case of the manifolds $W_{\mathrm{PL} 1,2}^{U}$ plotted in Figs. 8a,b, which are calculated from 21 iterations of an initial segment of length $d s=10^{-4}$ close to PL1 or PL2, we see that the manifolds fill only partially the chaotic domain up to the torus marked KAM. The inner dark region covered by the first iterations of the invariant manifolds defines a domain called "inner stickiness zone", the projection of which in the configuration space is shown schematically as a dark gray domain in Fig. 7c. The remaining part of the chaotic domain up to the curve marked "KAM" corresponds essentially to the light gray domain of Fig. 7c. This domain is eventually covered by the invariant manifolds after a very large number of iterations. For example, the manifolds of Figs. 8a,b have not yet reached the curve KAM after about 50 iterations, which in both models correspond to about 60 pattern rotation periods. Thus, during all this time interval the manifolds support a spiral structure.

The stickiness phenomena keep playing a significant role even when the spiral perturbation is pushed to untypically high values. For example, in model C, (Figs. 8c,d) the spiral strength is $Q_{\mathrm{s}}=0.57$, and under such a high value all the rotational KAM curves are destroyed, at least up to the outer Lindblad resonance. Then, while in principle there is no absolute barrier to chaotic diffusion up to very large distances from corotation, a plot of the invariant manifolds (Fig. 8c) shows that these manifolds exhibit again stickiness phenomena, and they practically remain confined for very large times below the $-4 / 1$ resonance (which is still stable at the value of the Jacobi constant $E_{\mathrm{J}}=-2.912 \times 10^{5}$, yielding four tiny islands embedded in the large chaotic sea of Fig. $8 \mathrm{c}$ near the level $p_{\theta}=1.6$. This results in that the manifolds in the configuration space (Fig. 8d) yield the form of tightly wound spiral arms. In this particular example, the domain covered by the invariant manifolds practically coincides with the "inner stickiness domain" of Fig. 7c.

\section{Spiral arms as the response of invariant manifolds to bars}

One immediate consequence of the analysis of the previous sections is that one cannot induce the morphology of the spiral arms, corresponding to a particular morphological type of bar, by calculating the invariant manifolds of the PL1 and PL2 families in only a pure bar potential. In fact $V_{\text {spiral }}$ is most important near 

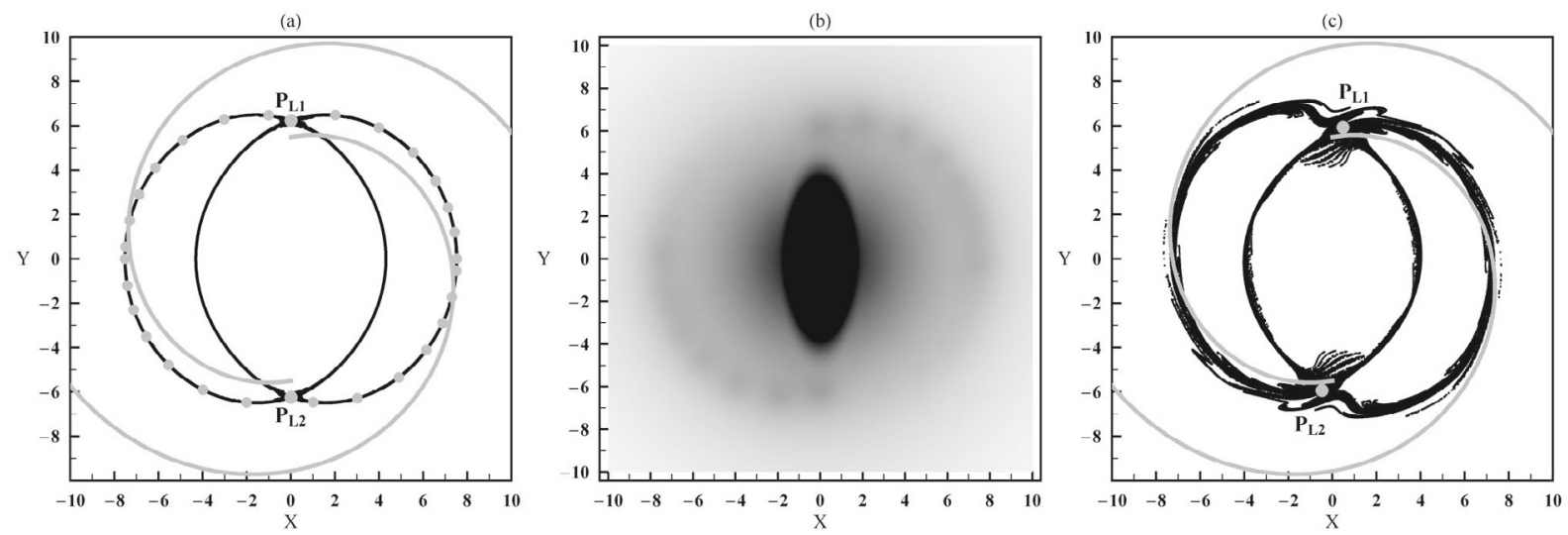

Fig. 9. A "spiral response" model based an initially "pure bar" version of model A. a) Manifolds of the pure bar case for $E_{\mathrm{J}}=-1.90 \times 10^{5}$ (very close to the $E_{\mathrm{J}, L 1}$ value. These manifolds are populated by "point masses" represented as Plummer spheres centered at the positions indicated by the the gray thick dots (see text for details). b) Gray scale mapping of the surface density produced by the mass distribution of a). c) Response manifolds calculated in the new potential corresponding to the mass distribution of a).

corotation and it must be taken into account self-consistently in all studies related to the morphology of the spiral arms via the calculation of invariant manifolds. This result is in agreement and probably provides a dynamical basis for understanding the results of both particle and hydrodynamical simulations (Lindblad et al. 1996; Aguerri et al. 2001) which have reported the inefficiency of simulations of pure bars to reproduce a spiral structure.

On the other hand, the theory of the invariant manifolds suggests that the spiral arms are linked dynamically to the bar. A plausible scenario for establishing such a link is one in which the bar initiates the process of a spiral response, which is then enhanced self-consistently by the growing contribution of the spiral potential.

The question addressed below is whether, on the sole knowledge of the gravitational potential and pattern speed of a pure bar model, such a process can be modeled via the theory of the invariant manifolds. We construct such an iterative "spiral response" model as follows:

i) we first calculate the invariant manifolds produced by the pure bar model;

ii) we assume that the invariant manifolds produced by the pure bar alone "trigger" the formation of a ring-like or spiral pattern by attracting matter along the invariant manifold. The density gradient along the manifold cannot be uniform, since (a) the speed of chaotic diffusion is smaller close to $L_{1}$ or $L_{2}$ than far from these points, and (b) the higher order lobes of the invariant manifolds return to the neighborhood of $L_{1}$ and $L_{2}$ (Sect. 2.2). In order to model the mass distribution along the invariant manifolds, we consider a number $N_{\mathrm{p}}$ of small Plummer spheres, of mass $m_{\mathrm{p}}$ and softening radius $r_{\mathrm{p}}$, placed along the invariant manifold of the pure bar case (Fig. 9a) with a linearly decreasing mass from $L_{1}$ or $L_{2}$ counterclockwise, namely the mass of the $i$ th particle is given by:

$m_{\mathrm{p}}(i)=\frac{2 M_{\mathrm{s}}}{N_{\mathrm{p}}}-\frac{2 M_{\mathrm{s}}}{\left(\frac{N_{\mathrm{p}}}{2}+1\right) N_{\mathrm{p}}} i$

with $i=1,2, \ldots, N_{\mathrm{p}} / 2$ from $L_{1}$ to $L_{2}$ counterclockwise and similarly along the symmetric manifold from $L_{2}$ to $L_{1}$. $M_{\mathrm{s}}$ is an estimate of the total mass on the spiral arms (see below). The choice of a linear mass decrease as in (16) along the response spirals is rather arbitrary and it does not follow directly from the theory of the invariant manifolds. However, it does capture the essential feature that the density of points should in general decrease along the unstable manifold as we recede from the unstable periodic orbit.

In the simulation of Fig. 9, we start from the invariant manifolds of the pure bar version of model A, and set $r_{\mathrm{p}}=0.5$, $N_{\mathrm{p}}=30$, and the mass of each particle fixed so that the total mass of all the particles is equal to $M_{\mathrm{s}}$ given by

$M_{\mathrm{S}}=\frac{1}{2} \int_{r_{1}}^{r_{2}} \int_{0}^{2 \pi} \int_{-\infty}^{\infty}\left|\rho_{\mathrm{s}}(r, \theta, z)\right| r \mathrm{~d} r \mathrm{~d} \theta \mathrm{d} z$

where the quantity $\rho_{\mathrm{s}}(r, \theta, z)$ is an approximate expression for the density perturbation corresponding to the potential (7) given by the WKB ansatz:

$\rho_{\mathrm{s}}(r, \theta, z)=-\frac{|k \Delta|(|k \Delta|+1)}{4 \pi G \Delta^{2}} V_{\mathrm{s}}(r, \theta) \operatorname{sech}^{2+|k \Delta|}(z / \Delta)$

where $k=2 /(r \tan i)$, according to the formula given by Vandervoort (see Contopoulos \& Grosbøl 1988; the used values of $\Delta$ are given in Table 1). Thus, $M_{\mathrm{s}}$ represents an estimate of the total mass contained in the spiral arms of model A. A gray scale plot of the total surface density corresponding to the above mass distribution is shown in Fig. 9b;

iii) the gravitational potential is calculated anew taking into account the "response" potential produced by the Plummer masses positioned as described in step (ii). This also yields a new position of the Lagrangian points $L_{1}, L_{2}$ as well as a new form of their invariant manifolds. The procedure should be repeated until convergence of the positions of the Lagrangian points and of their manifolds towards a final form is obtained. In practice, we find that just one iteration suffices to obtain invariant manifolds which fit the imposed spiral perturbation up to an azimuth $\left(\theta^{\prime} \simeq \pi / 2\right)$. In particular, the manifolds of Fig. 9c cover a much thicker radial domain than those of the initial "pure bar" model (Fig. 9a), and they also exhibit an azimuthal deformation following from the non-uniform distribution of mass shown in Fig. 9 b.

The present method can in principle be used to explore the sequence of different morphological types of spiral arms obtained by the method of the invariant manifolds applied to a family of pure bar potentials, when the bar parameters, i.e., bar strength and pattern speed are altered. Such a type of study was undertaken by Romero-Gomez et al. (2007), without taking, however, into account the effects of the self-consistent spiral response. For 

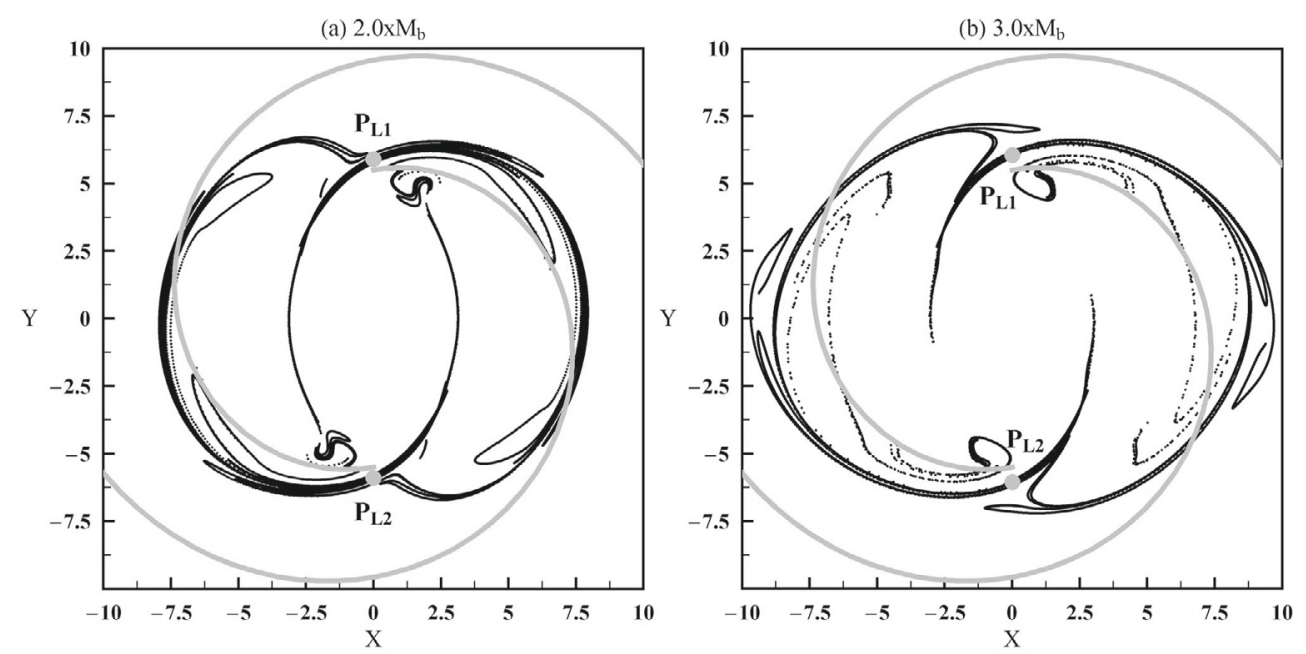

Fig. 10. Unstable invariant manifolds of the PL1 and PL2 orbits in a pure bar version of model A in which the bar's mass and pattern speed are altered with respect to the reference values $M_{\mathrm{b}}=1.5 \times 10^{10} M_{\odot}, \Omega_{\mathrm{p}}=43.6 \mathrm{~km} \mathrm{~s}^{-1} \mathrm{kpc}^{-1}$ so as to keep corotation at a fixed distance. a) $M_{\mathrm{b}}^{\prime}=2 M_{\mathrm{b}}$, $\Omega_{\mathrm{p}}^{\prime}=1.26 \Omega_{\mathrm{p}}, E_{\mathrm{J}}=-2.19 \times 10^{5}$. b) $M_{\mathrm{b}}^{\prime}=3 M_{\mathrm{b}}, \Omega_{\mathrm{p}}^{\prime}=1.38 \Omega_{\mathrm{p}}, E_{\mathrm{J}}=-2.40 \times 10^{5}$.

our adopted models and parameters, we find that when this effect is ignored, a kind of spiral response can only be produced by the low-order lobes of the invariant manifolds, but i) the bar strength has to be pushed to very high values; and ii) the spiral form disappears when the manifold is calculated for longer length. An example is given in Fig. 10, referring again to the "pure bar" version of model $\mathrm{A}$. The invariant manifolds are calculated after altering the bar parameters with respect to the values given in Kaufmann \& Contopoulos (1996). Figure 10 shows two different choices of parameters, in which the bar is given a mass equal to (a) two and (b) three times the mass value in Kaufmann \& Contopoulos (1996). The pattern speed was altered accordingly so as to keep corotation at a fixed distance. As the bar amplitude increases, the first lobes of the invariant manifolds become more open and they yield a gradual transition from a ring (Fig. 10a) to a spiral pattern (Fig. 10b). However, the spiral pattern in Fig. 10b does not fit the self-consistent spiral pattern of model A. Furthermore, the change of the bar strength required in order to produce this result constitutes a large deviation from the parameters of the original model which guaranteed self-consistency. This can be probably improved by choosing a lower $n$-value of the Ferrers bar and/or including the effects of a more rectangular bar outline.

A different methodology to produce response models of barred-spiral galaxies has been proposed by Patsis (2006). As already emphasized in Tsoutsis et al. (2008), the theory of the invariant manifolds explains many features of such response models. Patsis (2006) considers an ensemble of particles with initial conditions on circular orbits of the axisymmetric part of the potential, placed uniformly on the disk at radii corresponding to Jacobi constants up to the $L_{4}$ value of the full potential. Then, by softly introducing the non-axisymmetric part of the potential (the transition time is a few pattern periods), the particles' distribution changes due to both the adiabatic change of the potential and phase mixing. As a result, the particles finally settle to orbits supporting both the bar and the spiral arms.

Inside the bar, the particles of such response models are captured mostly in stable resonances belonging to a branch or bifurcation of the $x_{1}$ family, which is the continuation of the family of circular orbits of the axisymmetric potential on which all the initial conditions lie. However, near corotation and beyond chaos is prominent. In this case the theory of the invariant manifolds explains the capture of the particles in orbits supporting the spiral arms. In our specific models, one can see this effect by taking initial conditions $\left(\theta, p_{\theta}\right)$ on the surfaces of section such as those of Figs. 4a and 5a such that these initial conditions a) belong to a chaotic domain of their respective surfaces of section, and b) determine circular orbits when all the non-axisymmetric part of the potential is turned off (the monopole contribution of the bar, obtained by averaging radial forces with respect to all possible azimuths, is taken into account in this calculation). The locus of all these initial conditions is a straight line $p_{\theta}=$ const. on the surface of section for a given Jacobi constant. In practice, we take a narrow zone of some width around such a line, i.e., allow also for a small value of the epicyclic action around the circular orbits of the axisymmetric model.

Figure 11 shows the result of running these initial conditions in the case of model A, with the non-axisymmetric part of the potential being turned on from the start, and for one iteration (Figs. 11a,b, corresponding to 1.2 pattern rotation periods), or ten iterations (Figs. $11 \mathrm{c}, \mathrm{d}$, corresponding to 12 pattern rotation periods), for the value of the Jacobi constant $E_{\mathrm{J}}=-1.911 \times 10^{5}$. The initial conditions correspond to a zone in Fig. 11a between the two gray horizontal lines, that is we take 10000 points uniformly distributed within the intersection of the zone with the permissible domain of motion. The first iterates of these points are shown with dark thick points in the same figure, while the large number of small dots illustrate the overall structure of the phase portrait at the chosen value of $E_{\mathrm{J}}$. The corresponding figure in configuration plane is shown in Fig. 11b. An obvious conclusion from Figs. 11a,b is that, already after one iteration, the initially straight zone of initial conditions in the surface of section is deformed so as to closely follow a pattern induced essentially by the invariant manifolds $\mathcal{W}_{\mathrm{PL} 1,2}^{U}$ (compare Figs. 11a,b with Figs. 4a,b). This phenomenon is repeated at subsequent iterations, so that after ten iterations (Figs. 11c,d) the set of all points describes a pattern nearly coinciding with that of the manifolds $\mathcal{W}_{\mathrm{PL} 1,2}^{U}$, but over a larger length of the latter. This picture does not change qualitatively if the zone of initial conditions is taken to have a width four times as large as in Fig. 11a. The resulting response of the orbits in this case is shown in Fig. 11e, and the association of this with the dynamics of the invariant manifolds is still quite clear. 

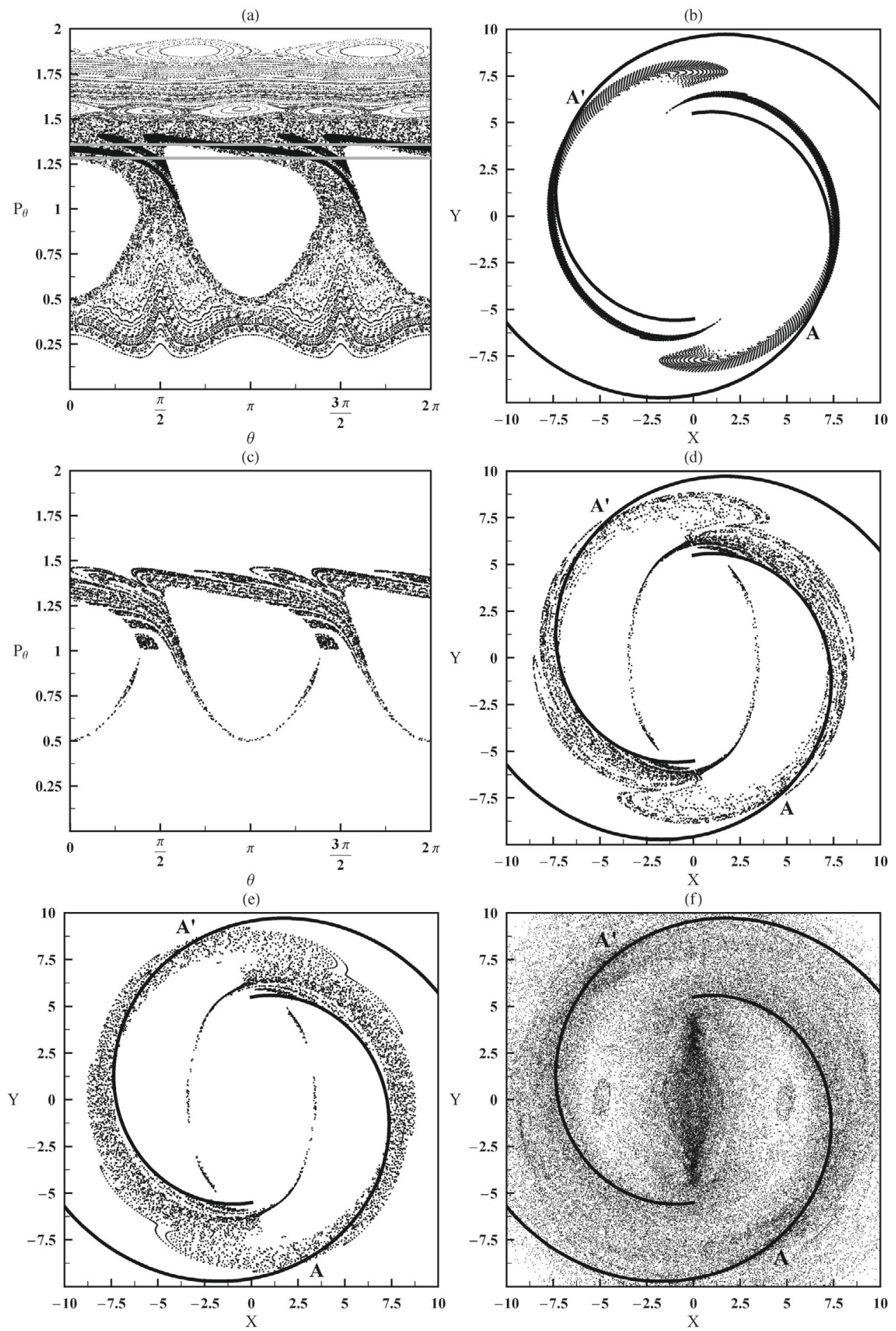

Fig. 11. Model A for the value of the Jacobi constant $E_{\mathrm{J}}=-1.911 \times 10^{5}$. a) The two gray horizontal lines define a zone of initial conditions around the circular orbit of the unperturbed (axisymmetric) potential. The thick black dots are the images of these initial conditions after one iteration of the Poincaré map. The background thin points show the underlying phase space structure. b) The first image of the Poincaré map of the initial conditions specified in a) as it appears in the configuration space. After only one iteration this set of points already acquires the form of the invariant manifolds, i.e., it yields trailing spiral arms. The projection of the tenth iterates of the same points on the surface of section and on the configuration space are shown in c) and d) respectively. e) Same as in d) when the initial conditions are taken in a zone four times as wide as the zone of a). f) A full "response model" for the same galaxy produced according to the methodology suggested in Patsis (2006). The black curves represent the minima of the imposed spiral potential.

This behavior follows from a well known "mixing property" of chaotic dynamical systems, namely that any small and compact ensemble of initial conditions embedded in a large chaotic domain is deformed in subsequent Poincaré mappings so as to follow the unstable invariant manifolds of the main families of unstable periodic orbits located in the chaotic domain, while preserving its measure in the same domain (see Contopoulos \& Harsoula 2008, and references therein for a detailed exploration 
of this phenomenon in the case of the "standard map"). In the case of barred galaxies, this type of response of the chaotic orbits to the invariant manifolds generates patterns such as those of Figs. 11b,d i.e., spiral arms.

It should be stressed, however, that Figs. $11 \mathrm{~b}$ to $11 \mathrm{e}$ were calculated with the full non-axisymmetric potential, i.e., $V_{\mathrm{bar}}+$ $V_{\text {spiral }}$, turned on from the start, i.e., without some initial transient time of growth of the non-axisymmetric part, and for just one value of the Jacobi constant. Figure 11f shows a "response model" obtained precisely via the method suggested by Patsis (2006). We thus take the initial conditions of $10^{5}$ particles on circular orbits of the axisymmetric part of the potential and on part of a uniformly populated disk, with distances in a range corresponding to the whole range of Jacobi constant values up to $E_{\mathrm{J}, L 4}$ of the full potential. The non-axisymmetric part of the potential was now introduced softly (in two pattern periods), and the particles were found to settle to a nearly invariant distribution in the configuration space after about 15 pattern periods. Figure 11f shows this distribution at a time corresponding to the 25th pattern period. Clearly, the particles have settled to orbits supporting both the bar and the imposed spiral arms. But the most interesting feature of this distribution is that the maxima of the density of the response spirals depart from the maxima of the imposed spiral at nearly the same points (points $A$ and $A^{\prime}$ ) where this happens for the invariant manifolds of Fig. 4b, which are also essentially traced by the points in Figs. 11b,d, or e. The interest of this result lies in that the initial conditions of the response model of Fig. 11f are selected from a nearly homogeneous distribution in space, and thus they are by no means associated with the invariant manifolds. In this respect, the invariant manifolds play for chaotic orbits a role similar to that of stable resonances for regular orbits, i.e. the manifolds are able to capture the chaotic orbits in their neighborhood and to create response spiral arms.

\section{Conclusions}

In the present paper we examined the applicability of the theory of the invariant manifolds emanating from the unstable short period orbits around the Lagrangian points $L_{1}$ and $L_{2}$ of a barred galaxy in the self-consistent models of Kaufmann \& Contopoulos 1996) which are rough models representing some features of real barred-spiral galaxies. We also tested the theory in weak or mean spiral versions of these models. Our conclusions are the following:

1. When both the bar and spiral components of the selfconsistent models are taken into account, the projection of the invariant manifolds on the configuration space produces a pattern that follows closely the imposed spiral pattern of the self-consistent model.

2. The addition of the spiral potential produces two effects: a) it shifts the position of the unstable Lagrangian points $L_{1}$, or $L_{2}$, both radially and angularly, and b) it enhances chaos locally, in a zone around corotation, due to the increase of the amplitude of the non-axisymmetric perturbation. The azimuthal displacement of the Lagrangian points is the most important factor for the manifolds to obtain a spiral form. In artificial models in which the whole non-axisymmetric perturbation is 'aligned' to the bar (e.g. by setting the phase of the spiral term equal to $\Phi=\theta$ ), the manifolds yield ring rather than spiral structures. If the amplitude of the perturbation is pushed to very high values, the lowest order lobes of the invariant manifolds can determine spiral patterns. However, we find that such patterns i) do not fit the spiral patterns of the self-consistent models; and ii) disappear after a few iterations of the calculation of the manifolds. This could probably be improved also by considering a lower $n$ value of the Ferrers bar, yielding a more gradual decrease of the bar force beyond the bar's end, and/or rendering the bar's outline more rectangular.

3. We construct a simple theoretical model yielding the boundaries of the apocenters of the chaotic orbits in the configuration space. Since the manifolds are developed within these boundaries, we demonstrate that they necessarily take the form of spiral arms, provided that the azimuths of the Lagrangian points $L_{1}, L_{2}$ are shifted with respect to the bar's major axis. The outermost radial limits of the manifolds are posed by rotational KAM curves outside corotation. However, in practice there are more stringent limits posed by the existence of cantori limiting the chaotic diffusion. The cantori define a narrow stickiness zone beyond corotation. The stickiness causes confinement of the invariant manifolds inside this zone for times comparable to the age of the galaxy.

4. A simple iterative method is proposed to calculate "spiral response" models on the basis of the theory of the invariant manifolds, starting from the invariant manifolds of a pure bar model. We also discuss the relevance of the response models of Patsis (2006) to the theory of the invariant manifolds. Following a general property of the Hamiltonian flow in a connected chaotic domain, a small sub-domain of initial conditions embedded within the chaotic domain yields successive Poincaré maps following closely the form of the invariant manifolds of the main families of unstable periodic orbits in the same domain. The consequences of this effect are tested by calculating a full response model via the method proposed by Patsis (2006). Despite the fact that the initial conditions of the particles in the latter model have no relevance to the invariant manifolds, the maxima of the response spiral arms in the final state are found to follow closely the underlying pattern formed by the invariant manifolds.

Acknowledgements. P. Tsoutsis and C. Kalapotharakos were supported in part by the Research Committee of the Academy of Athens. We thank an anonymous referee for the numerous comments which improved the paper.

\section{Appendix A: Permissible regions for motion}

The Hamiltonian (1), where $p_{r}=\dot{r}$ and $p_{\theta}=\dot{\theta}+\Omega_{\mathrm{p}} r^{2}$ defines a curve of zero velocity $\dot{r}=\dot{\theta}=0$ given by

$$
-\frac{1}{2} \Omega_{\mathrm{p}}^{2} r^{2}+V_{0}(r)+V_{1}(r, \theta)=E_{\mathrm{J}}
$$

where $V_{0}(r)$ and $V_{1}(r, \theta)$ are the total axisymmetric and nonaxisymmetric potential terms respectively. Denoting by $r_{\mathrm{s}}$ the corotation distance when only axisymmetric potential terms are taken into account, and $V_{\mathrm{s}} \equiv V_{0}\left(r_{\mathrm{s}}\right)$, we have $\Omega_{\mathrm{p}}^{2}=V_{\mathrm{s}}^{\prime} / r_{\mathrm{s}}$. The curves of zero velocity around $L_{4}$ and $L_{5}$ can be found by expanding the radius and the Jacobi constant with respect to the corotation values, $r=r_{\mathrm{s}}+\left(r-r_{\mathrm{s}}\right), E_{\mathrm{J}}=-\frac{\Omega^{2} r_{\mathrm{s}}^{2}}{2}+V_{\mathrm{s}}+h$. We find:

$2 h=-M\left(r-r_{\mathrm{s}}\right)^{2}+\frac{V_{\mathrm{s}}^{\prime \prime}}{3}\left(r-r_{\mathrm{s}}\right)^{3}+\ldots+2 V_{1}(r, \theta)$

where $M=\Omega_{\mathrm{p}}^{2}-V_{0}^{\prime \prime}=4 \Omega_{\mathrm{p}}^{2}-\kappa_{\mathrm{s}}^{2}\left(\kappa_{\mathrm{s}}\right.$ denotes the epicyclic frequency).

Consider a very simple form for $V_{1}$, e.g. $V_{1}=A \cos (2 \theta)$. For all values of the Jacobi constant between $E_{\mathrm{J}}=E_{\mathrm{J}, L_{1}}$ 
and $E_{\mathrm{J}}=E_{\mathrm{J}, L_{4}}$ we have $h-A<0$, thus, since $r-r_{\mathrm{s}}$ is small, we find from (A.2) that $M>0$, or $2 \Omega_{\mathrm{p}} / \kappa_{\mathrm{s}}>1$. Omitting terms of third order in $\left(r-r_{\mathrm{s}}\right)$, we find from Eq. (A.2)

$r-r_{\mathrm{s}}= \pm\{[2 A \cos (2 \theta)-2 h] / M\}^{1 / 2}$.

If $-A<h<A$ the motions are outside two banana-like curves around $L_{4}$ and $L_{5}$. In particular, for $r=r_{\mathrm{s}}$ we have, around $L_{4}$, $\theta= \pm \frac{1}{2} \cos ^{-1}(h / A)$, while for $\theta=0$ we have

$r-r_{\mathrm{s}} \simeq \pm[2(A-h) / M]^{1 / 2}$.

Equation (A.4) gives the intersections of the banana-like curves of zero velocity on the axis connecting the center to the stable Lagrangian points. The forbidden regions of the CZV can be compared with the corresponding forbidden regions of the surface of section $\dot{r}=0$, which is given by Eq. (15). In the latter case we have $p_{\theta}^{2}=r V^{\prime}=\Omega^{2} r^{2}, \Omega^{2} r^{2}-2 \Omega \Omega_{\mathrm{p}} r^{2}+2\left(V_{0}+V_{1}-E_{\mathrm{J}}\right)=0$. Developing $r, \Omega$ and $V_{0}$ in powers of $r-r_{\mathrm{s}}$ and omitting terms of order higher than the second we find the boundary of the forbidden region:

$\left(-M+\frac{M^{2}}{4 \Omega_{\mathrm{p}}^{2}}\right)\left(r-r_{\mathrm{s}}\right)^{2}+2 A \cos (2 \theta)=2 h$.

This gives

$r-r_{\mathrm{s}}= \pm[2 A \cos (2 \theta)-2 h]^{1 / 2} \frac{2 \Omega_{\mathrm{p}}}{\kappa_{\mathrm{s}}}$.

Since $2 \Omega_{\mathrm{p}} / \kappa_{\mathrm{S}}>1$, this boundary is larger than the boundary (A.3).

\section{References}

Aguerri, J. A. L., Hunter, J. H., Prieto, M., et al. 2001, A\&A, 373, 786 Buta, R., Vasylyev, S., Salo, H., \& Laurikainen, E. 2005, AJ, 130, 506 Contopoulos, G. 1981, A\&A, 102, 265

Contopoulos, G. 2002, Order and Chaos in Dynamical Astronomy (Berlin: Springer-Verlag)

Contopoulos, G., \& Grosbøl, P. 1988, A\&A, 197, 83

Contopoulos, G., \& Harsoula, M. 2008, Int. J. Bif. Chaos, 18, 1

Contopoulos, G., Harsoula, M., Voglis, N., \& Dvorak, R. 1999, J. Phys. A: Math. Gen., 32, 5213

Efthymiopoulos, C., Contopoulos, G., Voglis, N., \& Dvorak, R. 1997, J. Phys. A: Math. Gen., 30, 8167

Fux, R. 2001, A\&A, 373, 511

Grobman, D. M. 1959, Dokl. Akad. Nauk SSSR, 128, 880

Grosbøl, P. 2003, in Galaxies and Chaos, ed. G. Contopoulos, \& N. Voglis, Lect. Notes Phys., 626, 201

Hammed, S., \& Devereux, N. 1999, ApJ, 118, 730

Hartman, P. 1960, Proc. Amer. Math. Soc., 11, 610

Kaufmann, D. E., \& Contopoulos, G. 1996, A\&A, 309, 381

Kaufmann, D. E., \& Patsis, P. 2005, ApJ, 624, 693

Laurikainen, E., \& Salo, H. 2002, MNRAS, 337, 1118

Lindblad, P. A. B., Lindblad, P. O., \& Athanassoula, E. 1996, A\&A, 313, 65

Patsis, P. A. 2006, MNRAS, 369, 56

Patsis, P. A., Efthymiopoulos, C., Contopoulos, G., \& Voglis, N. 1997, A\&A, 326, 493

Pfenniger, D. 1984, A\&A, 134, 373

Pfenniger, D., \& Friedli, D. 1991, A\&A, 252, 75

Pichardo, B., Martos, M., \& Moreno, E. 2004, ApJ, 609, 144

Romero-Gomez, M., Masdemont, J. J., Athanassoula, E. M., \& Garcia-Gomez, C. $2006, A \& A, 453,39$

Romero-Gomez, M., Athanassoula, E. M., Masdemont, J. J., \& Garcia-Gomez, C. $2007, A \& A, 472,63$

Schwarzschild, M. 1979, ApJ, 232, 236

Smith, B., Miller, R. 1982, ApJ, 257, 103

Sparke, L. S., \& Sellwood, J. A. 1987, MNRAS, 225, 653

Tsoutsis, P., Efthymiopoulos, C., \& Voglis, N. 2008, MNRAS, 387, 1264

Tully, R. B., Verheijen, M. A. W., Pierce, M. J., Huang, J.-S., \& Wainscoat, R. J. 1996, ApJ, 112, 2471

Voglis, N., Tsoutsis, P., \& Efthymiopoulos, C. 2006a, MNRAS, 373, 280

Voglis, N., Stavropoulos, I., \& Kalapotharakos, C. 2006b, MNRAS, 372, 901 